\title{
SELENOPROTEIN O is a chloroplast protein involved in ROS scavenging and its absence increases dehydration tolerance in Arabidopsis thaliana
}

\author{
Yosef Fichman ${ }^{\mathrm{a}}$, Zsuzsa Koncz ${ }^{\mathrm{b}}$, Noam Reznik ${ }^{\mathrm{a}}$, Gad Miller ${ }^{\mathrm{c}}$, László Szabados ${ }^{\mathrm{d}}$, \\ Katharina Kramer ${ }^{\mathrm{e}}$, Hirofumi Nakagami ${ }^{\mathrm{e}}$, Hillel Fromm ${ }^{\mathrm{a}, 1}$, Csaba Koncz ${ }^{\mathrm{b}, \mathrm{d}, 1}$, Aviah Zilberstein ${ }^{\mathrm{a}, *, 1}$ \\ ${ }^{a}$ School of Plant Sciences and Food Security, Tel Aviv University, P.O. Box 39040, Tel Aviv 6997801, Israel \\ b Department of Plant Developmental Biology, Max-Planck Institute for Plant Breeding Research, Carl-von-Linné-Weg 10, D-50829 Cologne, Germany \\ ${ }^{\mathrm{c}}$ The Mina and Everard Goodman Faculty of Life Sciences, Bar-Ilan University, Ramat-Gan 5290002, Israel \\ d Institute of Plant Biology, Biological Research Center of Hungarian Academy of Sciences, Temesvári krt. 62/64, H-6724 Szeged, Hungary \\ e Protein Mass Spectrometry Group, Max-Planck Institute for Plant Breeding Research, Carl-von-Linné-Weg 10, D-50829 Cologne, Germany
}

\section{A R T I C L E I N F O}

\section{Keywords:}

Selenoprotein $\mathrm{O}$

ROS

Drought tolerance

Proline metabolism

Abiotic stress signaling

\begin{abstract}
A B S T R A C T
The evolutionary conserved family of Selenoproteins performs redox-regulatory functions in bacteria, archaea and eukaryotes. Among them, members of the SELENOPROTEIN O (SELO) subfamily are located in mammalian and yeast mitochondria, but their functions are thus far enigmatic. Screening of T-DNA knockout mutants for resistance to the proline analogue thioproline (T4C), identified mutant alleles of the plant SELO homologue in Arabidopsis thaliana. Absence of SELO resulted in a stress-induced transcriptional activation instead of silencing of mitochondrial proline dehydrogenase, and also high elevation of $\Delta(1)$-pyrroline-5-carboxylate dehydrogenase involved in degradation of proline, thereby alleviating T4C inhibition and lessening drought-induced proline accumulation. Unlike its animal homologues, SELO was localized to chloroplasts of plants ectopically expressing SELO-GFP. The protein was co-fractionated with thylakoid membrane complexes, and co-immunoprecipitated with FNR, PGRL1 and STN7, all involved in regulating PSI and downstream electron flow. The selo mutants displayed extended survival under dehydration, accompanied by longer photosynthetic activity, compared with wild-type plants. Enhanced expression of genes encoding ROS scavenging enzymes in the unstressed selo mutant correlated with higher oxidant scavenging capacity and reduced methyl viologen damage. The study elucidates SELO as a PSI-related component involved in regulating ROS levels and stress responses.
\end{abstract}

\section{Introduction}

Plants respond to environmental changes by altering many molecular and physiological processes. These responses are tightly controlled at cellular, organ and whole plant levels. Key components in this interplay are redox regulation and generation of reactive oxygen species (ROS) [1]. Changes in redox homeostasis affect protein structure and enzymatic activity, and influence signal transduction [2]. In plants, most of ROS production is modulated by changes in the activity of electron transport chains in chloroplasts and mitochondria [3]. ROS act as secondary messengers mediating stress responses while their levels are tightly controlled to prevent cellular damage [4]. ROS scavenging comprises a large part of cellular responses to abiotic stresses.
Scavengers include specific enzymes that reduce free radicals to water in multi-stage reactions [5], and anti-oxidative metabolites [6]. Proline (Pro) is a unique metabolite, which usually accumulates in response to different abiotic and biotic stresses, mostly due to stress-induced changes in the transcription of genes that encode enzymes of the evolutionary conserved glutamate (Glu)-proline-glutamate cycle [7-12]. In mammals and plants, Pro catabolism to Glu occurs in mitochondria concomitantly delivering electrons to the mitochondrial electron transport chain (ETC). Intensive oxidation of Pro to P5C in mitochondria increases electron flux and elevates ROS generation $[8,10]$. Although Pro accumulates in plant cells under stress conditions [13,14], its protective role in overcoming stress damages is still under debate [14-18].

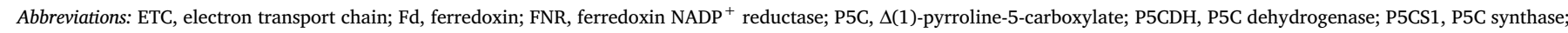

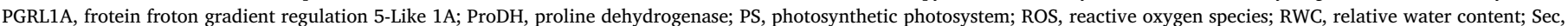
selenocysteine; SELO, selenoprotein O; STN7, regulatory serine/threonine-protein kinase 7; T4C, L-thiazolidine-4-carboxylate ( $\gamma$-thioproline)

* Corresponding author.

E-mail address: aviah@post.tau.ac.il (A. Zilberstein).

${ }^{1}$ Authors with equal contribution. 
In a forward genetic screen, we isolated Arabidopsis thaliana T-DNA knockout mutants [19] capable of growing in the presence of lethal concentration of the Pro analogue L-thiazolidine-4-carboxylate (T4C, $\gamma$ thioproline) in $\mathrm{NaCl}$-containing medium [8]. In Arabidopsis, salt-stress and dehydration-induced signaling elevate cellular Pro synthesis and simultaneously prevent mitochondrial Pro degradation by blocking the transcription of proline dehydrogenase 1 (ProDH1) leading to Pro accumulation [17]. Reduced ProDH1 activity during stress inhibits Pro oxidation to $\Delta(1)$-pyrroline-5-carboxylate (P5C) and also T4C breakdown. T4C competes with Pro incorporation during translation causing protein disfunctioning, and blocking cell division and growth. Thus, T4C-tolerant mutants are expected to be impaired in normal stress-induced down-regulation of ProDH1 essential for Pro catabolism [8].

One of the T4C tolerant mutants, displaying higher tolerance to dehydration, carried a T-DNA insertion in the Arabidopsis gene AT5G13030 encoding a plant homologue of SELENOPROTEIN O (SELO). Selenoproteins contain selenocysteine (Sec) in their redox motifs [20]. Such substitution of sulfur atom with selenium in the cysteine residue increases nucleophilicity and redox activity. Conserved selenoprotein families with known functions include glutathione peroxidases [21], thioredoxin reductases [22] and deiodinases [23]. As plants lack selenocysteine-tRNA, their selenoprotein homologues contain cysteine residues instead of Sec in the redox motifs [20,24]. Human SELO is located in mitochondria and possesses a C-terminal CxxSecSS redox motif, which catalyzes the formation of complexes through S-S and S-Se bridges with yet unknown protein partner(s), under oxidative conditions. Such redox-dependent complexes are also formed upon changing the motif to SxxSecSS, CxxCSS and SxxCSS [25], suggesting that the corresponding C-terminal CxxsCSS motif in plant SELO homologues likely maintains a similar redox activity. All members of the remarkably conserved SELO family contain a putative ATP-binding Walker-motive and some internal domains showing distant structural relationship to catalytic domains of porcine protein kinase A (PKA), including the archetypical His-Arg-Asp catalytic motif [26]. Nonetheless, the function of SELO, as well as its redox partner(s), remained so far unexplored.

Characterization of Arabidopsis selo mutation and its physiological effects in this study provides the first insight into the function of SELENOPROTEIN O in plants. SELO was localized to the chloroplast by using SELO-GFP fusion, and by its co-migration with thylakoid complexes on Blue-Native gels. Co-immunoprecipitation of proteins from isolated chloroplasts showed that SELO-GFP could interact with Ferredoxin NADP Reductase (FNR) and Protein Proton Gradient Regulation 5-Like 1A (PGRL1A), which are involved in electron transport from Photosystem I (PSI) [27], and also with the regulatory serine/ threonine-protein kinase STN7. Lack of SELO in the selo mutant affected the redox poise, maintained in WT plants under normal growth conditions, by reducing $\mathrm{H}_{2} \mathrm{O}_{2}$ levels and enhancing expression of genes coding for ROS scavenging enzymes, mostly active in chloroplasts. A change in plant response to dehydration was also observed. The selo mutants were more tolerant to dehydration and their photosynthetic activity was extended compared with that of WT plants. Furthermore, regular signaling of Pro accumulation in response to stress was impaired in selo mutants leading to transcriptional induction, instead of silencing, of genes encoding enzymes of mitochondrial Pro catabolism during dehydration. To this end, our data indicate that SELENOPROTEIN O is involved in mediating ROS levels, mostly in chloroplasts and thereby affecting downstream signaling and stress responses.

\section{Materials and methods}

\subsection{Plant material, growth conditions, and stress induction}

Arabidopsis thaliana, ecotype Columbia (Col-0), and its T-DNA insertion mutants were used in all experiments. The selo1-1 mutant was identified in the T-DNA-knockout mutant collection of Alvarado et al.
[19], whereas the selo1-2 (GABI_956D07; [28]) and selo1-3 (SAIL_776_G08; [29]) mutants were obtained from the Arabidopsis Biological Resource Center (ABRC). Overexpression lines were generated using the ABRC cDNA stock GC105358 [30]. Plants were grown in growth chamber in pots under $16 \mathrm{~h}$ dark $/ 8 \mathrm{~h}$ light regime $\left(100 \mu\right.$ mole $\left.\mathrm{m}^{-2} \mathrm{~s}^{-1}\right)$ at $22.5^{\circ} \mathrm{C}$ and $60 \%$ RH. Dehydration stress was imposed by stopping irrigation of 30-day-old plants, grown in pots containing equal soil weight. White light stress of $200 \mu \mathrm{mole} \mathrm{m}^{-2} \mathrm{~s}^{-1}$ photon flux was applied for 30 minutes. Oxidative stress was imposed by applying $0.25 \mu \mathrm{M}$ methyl viologen (Sigma-Aldrich, Merck) to 7-dayold seedlings, grown in $0.5 \mathrm{MS}$ medium for $48 \mathrm{~h}$ in wells of micro-titer plates (Ducefa Biochemie), without sucrose, using light intensity of $100 \mu$ mole $\mathrm{m}^{-2} \mathrm{~s}^{-1}$. Stress experiments were repeated three times with 15-45 replicates.

\subsection{Generation of overexpression lines}

SELO cDNA was cloned using EcoRI sites in pART7-GFP downstream to the CaMV 35S promoter, and then the expression cassette was shifted to the NotI site of the binary vector pART27 [31] yielding pART27SELO-GFP. The plasmid was introduced into the Agrobacterium tumefaciens GV3101 (pMP90RK) strain [32]. Floral dipping was used to transform Arabidopsis plants [33]. T1 seedlings were selected on MS containing $50 \mu \mathrm{g} / \mathrm{ml}$ kanamycin followed by screening eGFP fluorescence (ex. $488 \mathrm{~nm}$, em.560 nm) using Zeiss CLSM780 confocal microscope. The T-DNA of pART27-SELO-GFP construct was introduced into Arabidopsis overexpressing tpFNR-YFP, which was received from $\mathrm{J}$. Mathur (University of Guelph, Guelph, Ontario, Canada). These plants were obtained by introducing the CaMV35S promoter-tpFNR:YFP expression cassette cloned in the binary pCAMBIA vector, and applying selection for hygromycin resistance. Similar construct, containing tpFNR-mEosFP, was described by Schattat et al. [34]. Plants expressing SELO-GFP and tpFNR-YFP were selected on kanamycin and hygromycin containing MS medium.

\subsection{Physiological measurements}

Wilting was assessed by visualization of turgor loss of $50 \%$ of the rosette leaves. Relative water content [35] was calculated according to the equation of RWC $(\%)=[(W-D W) /(T W-D W)] \times 100$, by measuring fresh weight of excised leaves (W); turgid weight after dipping in $\mathrm{H}_{2} \mathrm{O}$ for $4 \mathrm{~h}$ (TW), and dry weight after overnight drying at $80^{\circ} \mathrm{C}$ (DW). Photosynthetic measurements were performed after $2 \mathrm{~h}$ of daily illumination (at $10 \mathrm{AM}$ ) by using the PlantScreen High-Through-put Phenotyping system of Photon Systems Instruments as described by Awlia et al. [36]. Measurements of PSII quantum yield (Fv/Fm), and electron flow rate (ETR) were conducted as described by Rungrat et al. [37]. Chlorophyll Fluorescence decrease ratio $\left(\mathrm{R}_{\mathrm{Fd}}\right)$ was estimated according to Lichtenthaler et al. [38] based on fluorescence values measured according to Rungrat et al. [37]. Stomatal density was determined using epidermis peel microscopy [39]. Stomata conductance was measured in 16 replicates by leaf porometer (Decagon Devices, Inc.) $2 \mathrm{~h}$ after starting daily illumination.

\section{4. qRT-PCR measurements}

RNA was extracted from leaves, two hours after starting daily illumination, using Plant RNeasy kit (Qiagen), and used at concentration of $200 \mathrm{ng}$ per reaction for cDNA synthesis by the High-Capacity cDNA Reverse Transcription Kit (Thermo Fisher Scientific). Quantitative polymerase chain reaction (qRT-PCR) was performed with primers listed in Table A1 and Fast SYBR Green Master mix (Thermo Fisher Scientific), using the StepOnePlus Real-Time PCR instrument (Thermo Fisher Scientific). Each type of reaction was performed with 3 biological replicates and 3 technical replicates. Results were analyzed with StepOne software to compare relative quantity of $2^{-\Delta \Delta \mathrm{Ct}}$ [40]. Results 
were calculated relative to expression of cyclophilin (AT2G36130) as a constitutively expressed control and WT values obtained under unstressed conditions.

\subsection{Measurements of Pro, ROS content in guard cell chloroplasts or in seedlings, and antioxidant capacity}

Pro was extracted from leaves and measured according to Bates et al. [41] and Miller et al. [8]. ROS measurements in guard cells were performed according to Leshem et al. [42]. Leaves were incubated with $5 \mu \mathrm{M} 2^{\prime}, 7^{\prime}$-Dichlorofluorescin diacetate (DCF, Sigma-Aldrich, Merck) in $\mathrm{EtOH} /$ water for $5 \mathrm{~min}$. Following washing with water for $5 \mathrm{~min}$, prepared slides were visualized by CLSM 780 (ex. $490 \mathrm{~nm}$, em. $520 \mathrm{~nm}$ ). Chloroplast fluorescence data were analyzed according to McCloy et al. [43] using ImageJ. To quantify $\mathrm{H}_{2} \mathrm{O}_{2}$ in seedlings, supernatants from $100 \mathrm{mg}$ extract samples of 7-day-old seedlings ground in $50 \mathrm{mM} \mathrm{Na}$ Phosphate buffer ( $\mathrm{pH}$ 7.4) were incubated with equal volume of $100 \mu \mathrm{M}$ 10-acetyl-3,7-dihydroxyphenoxazine (ADHP, Sigma-Aldrich, Merck) and $0.2 \mathrm{U} / \mathrm{mL}$ HRP (Sigma-Aldrich, Merck) for $30 \mathrm{~min}$. Fluorescence was estimated using plate reader (Biotek Synergy, ex. $571 \mathrm{~nm}$, em. $585 \mathrm{~nm}$ ), and ROS content was calculated according to standard curve of known concentrations of $\mathrm{H}_{2} \mathrm{O}_{2}$ [44]. For estimation of antioxidant capacity using TEAC [45], $10 \mu \mathrm{l}$ samples of ground leaf extracts $(0.1 \mathrm{gr}$ FW in $0.2 \mathrm{M} \mathrm{Na}$-Acetate buffer, $\mathrm{pH} 4.3$ ), or $10 \mu \mathrm{l} \mathrm{mM}$ Trolox (6-Hydroxy-2,5,7,8-tetramethylchromane-2-carboxylic acid, Sigma-Aldrich,

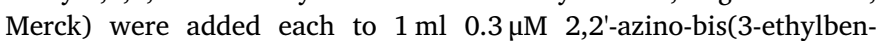
zothiazoline-6-sulphonic acid; ABTS), $0.15 \mu \mathrm{M}$ potassium persulfate (Sigma-Aldrich, Merck), and incubated for $15 \mathrm{~min}$. Antioxidants from the extracts or Trolox converted the colored ABTS radical to its colorless neutral form and changed the solution absorption at $734 \mathrm{~nm}$ (Ultrospec 2000 UV/VIS Spectrophotometer, GE Healthcare). The extent of ABTS radical elimination in the reactions was extrapolated to Trolox equivalents. Each experiment was repeated 3 times and included 5-10 replicates.

\subsection{Chloroplast isolation and immunoblotting}

Chloroplast isolation was carried out according to Joly and Carpentier [46]. Leaves ( $1 \mathrm{~g}$ fresh weight) were ground using Minilys personal homogenizer (Bertin technologies) with $5 \mathrm{~mL}$ buffer $(50 \mathrm{mM}$ HEPES pH 6.9, $0.33 \mathrm{M}$ sorbitol, $2 \mathrm{mM}$ EDTA, $1 \mathrm{mM} \mathrm{MgCl}_{2}, 1 \mathrm{mM}$ $\mathrm{MnCl}_{2}$ ). Following centrifugation ( $20000 \mathrm{xg}, 10 \mathrm{~min}$ ) chloroplast pellet was resuspended in $50 \mu 1$ 1\% Triton-X100, $750 \mathrm{mM}$ 6-aminocupronic acid and $0.2 \%$ dodecylmaltoside and incubated for $1 \mathrm{~h}$ at $4{ }^{\circ} \mathrm{C}$ for partial complex solubilization. Samples $(1 \mu \mathrm{g} / \mu \mathrm{l})$ were then resolved by SDSPAGE and analyzed by western blotting. Blue Native (BN) gel separation was performed according to Wittig et al. [47]. In brief, partially solubilized chloroplast complexes were loaded on a non-denaturing 4$16 \%$ polyacrylamide gradient gel ( $250 \mu \mathrm{g}$ protein in each lane). Separated proteins were then blotted onto PVDF membrane for immunodetection. Alternatively, bands were cut out from the gel for proteomics analysis. For immunodetection the membranes were incubated overnight with anti-GFP (BioLegend \#902601, 1:1000), antiTUBULIN (Sigma-Aldrich, Merck \#T9026, 1:500) or anti-RbcL ([48], 1:500) antibodies. HRP-conjugated goat anti-mouse (Jackson ImmunoResearch Laboratories \#115-035-003) secondary antibodies were used for GFP and TUBULIN detection, whereas anti-RbcL was probed with goat anti-rabbit (Jackson ImmunoResearch Laboratories \#111035-003) antibodies using ECL protein detection kit (Cyanagen).

\subsection{Identification of SELO protein interactors by Co-immunoprecipitation}

Aliquots of frozen chloroplasts were centrifuged and solubilized for 1 hour in $50 \mu 150 \mathrm{mM} \mathrm{NaCl}, 50 \mathrm{mM}$ imidazole, $2 \mathrm{mM}$ 6-Aminhexanoic acid, $1 \mathrm{mM}$ EDTA, pH 7. Each sample containing $300 \mu \mathrm{g}$ chlorophyll was incubated in $950 \mu \mathrm{l}$ binding buffer ( $50 \mathrm{mM}$ Na-phosphate buffer,
$\mathrm{pH} 7,50 \mathrm{mM} \mathrm{NaCl}$ ) with $8 \mu \mathrm{l}$ GFP-Trap agarose beads (Chromotek) overnight at $4{ }^{\circ} \mathrm{C}$ on a roller. After centrifugation at $2500 \mathrm{~g}$, beads were washed with $1 \mathrm{ml}$ binding buffer by rolling for 5 minutes at $4{ }^{\circ} \mathrm{C}$ and then centrifugation. The washing step was repeated twice, each for 10 minutes. Proteins were then eluted by boiling at $100{ }^{\circ} \mathrm{C}$ for $10 \mathrm{~min}$ utes with X2 Sample Buffer [4\% SDS, $120 \mathrm{mM}$ Tris-HCl pH 6.8, 0.04\% bromophenol blue (w/v), $0.2 \mathrm{M}$ Dithiothreitol]. Tubes were centrifuged at $4{ }^{\circ} \mathrm{C}$ for 10 minutes. Supernatant samples $(20 \mu \mathrm{l})$ were separated by SDS-PAGE. For immunodetection of pulled-down proteins, the western blot was probed with rabbit anti-FNR, anti-PGRL1A, or anti-STN7 antibodies as described in section 2.6.

\subsection{Confocal microscopy}

Cellular localization studies were performed using CLSM780 (Zeiss) confocal microscope. Each fluorophore was examined separately. Excitation was separately performed with $2 \%$ laser at $633 \mathrm{~nm}$ for chlorophyll auto-fluorescence, $2 \%$ laser at $514 \mathrm{~nm}$ for tpFNR-YFP, and $2 \%$ laser at $488 \mathrm{~nm}$ for SELO-GFP. The settings of emission filters were 639-735 nm for chlorophyll, 517-623 nm for YFP and 493-562 nm for GFP. Co-localization was calculated by Pearson correlation coefficient using the Coloc 2 algorithm (https://imagej.net/Coloc_2) in ImageJ [49].

\subsection{Proteomics analysis}

Bands of chloroplast complexes identified in BN gels were excised, and digested with trypsin. Peptides were analyzed by LC-MS/MS microsequencing on LTQ-Orbitrap (Thermo). Peptides were identified by Compound Discoverer software with two search algorithms, Sequest (Thermo) and Mascot (Matrix science), which were used to identify hits in the Arabidopsis thaliana Uniprot database [50], and decoy database (for determining the false discovery rate). All identified peptides were filtered with high confidence, top rank, mass accuracy, and a minimum of 2 peptides per protein. The analysis was performed by the Smoler Proteomics Center in the Technion (Haifa 32000, Israel).

\subsection{Bioinformatics analyses}

Multiple sequence alignments were performed using the BLOSUM62 algorithm in MAFFT [51]. Phylogenetic analysis was carried out in the Randomized Axelerated Maximum Likelihood program (RAxML; [52]). SELO gene expression data were collected from the eFP site [53] derived from the Affymetrix ATH1 array data [54-56]. Transcription data of Arabidopsis selenoprotein-like genes were obtained from the Genevestigator site [57].

\section{Results}

\subsection{Identification of selo knockout mutants and their physiological characterization}

Using a collection of about 20,000 Arabidopsis T-DNA-tagged lines transformed with the promoter trap vector pTluc [19], we performed a forward genetic screen to identify mutants capable of growing in the presence of $3 \mathrm{mM}$ T4C (Pro analogue) and $50 \mathrm{mM} \mathrm{NaCl}$. We have previously demonstrated that salt-treatment inhibits the transcription of mitochondrial proline dehydrogenase (ProDH1), enhances Pro accumulation and ROS production, and prevents degradation of T4C that competes with Pro in the translation process, leading to growth arrest [8]. Thus, the applied selection aimed at the isolation of mutations, which could relieve the inhibition of Pro/T4C degradation under saltstress. In one of the T4C tolerant lines, we identified a T-DNA insertion (selo1-1, Fig. 1A) located in the second exon of the gene AT5G13030 encoding a homologue of SELENOPROTEIN O. We obtained two other knockout alleles (selo1-2, GABI_956D07, and selo1-3, SAIL_776_G08) 
A

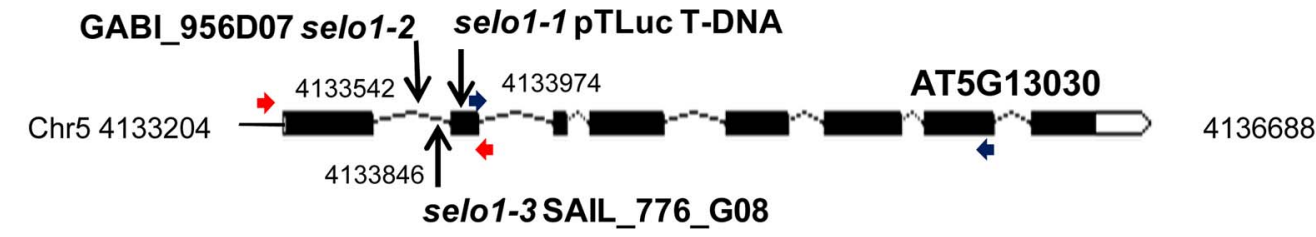

B

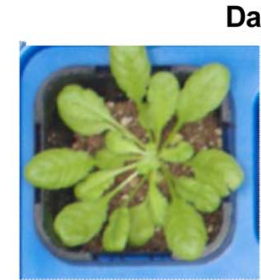

WT
Day 2

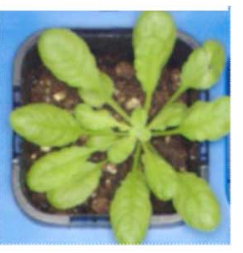

selo1-2

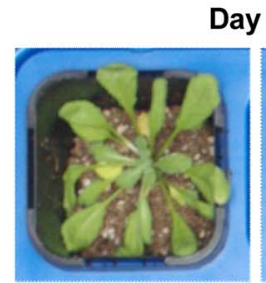

WT

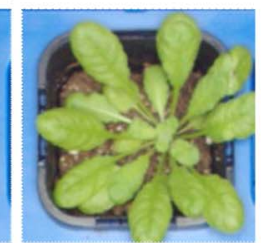

selo1-2
C
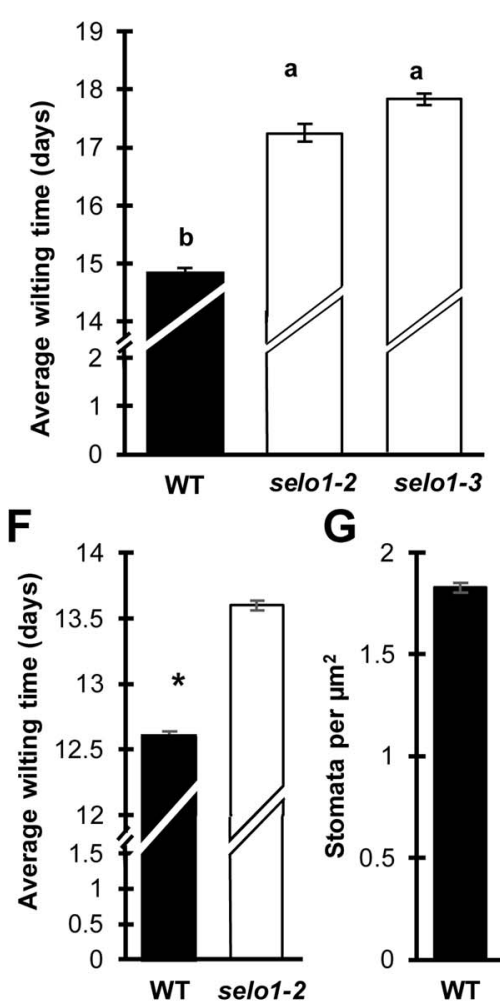

G
D
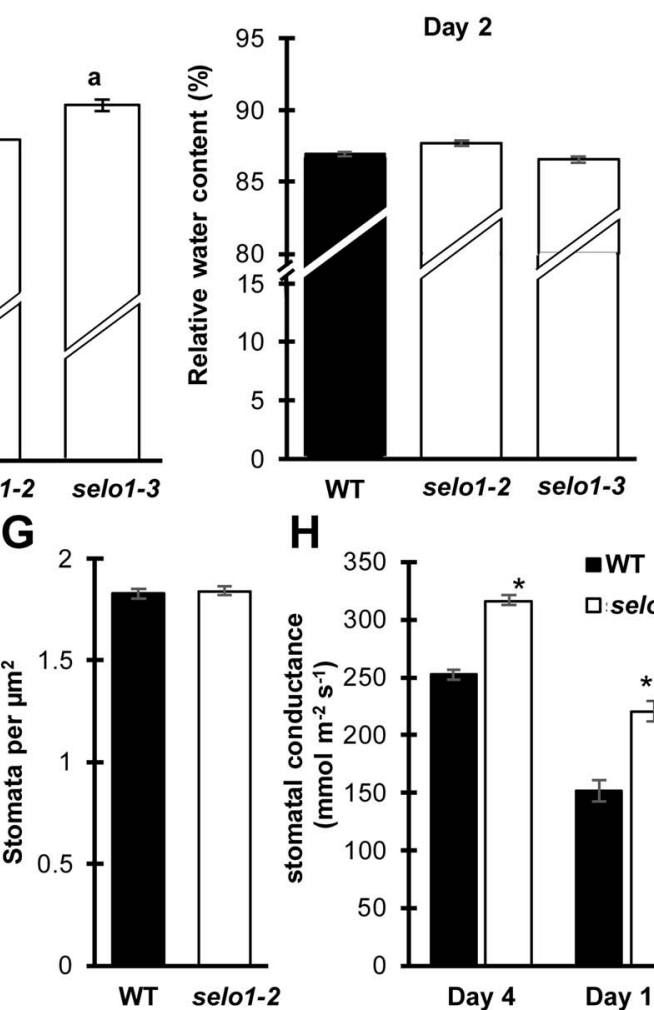

$\mathrm{H}$

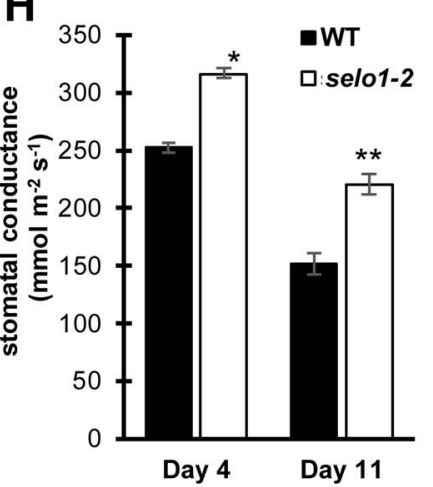

E

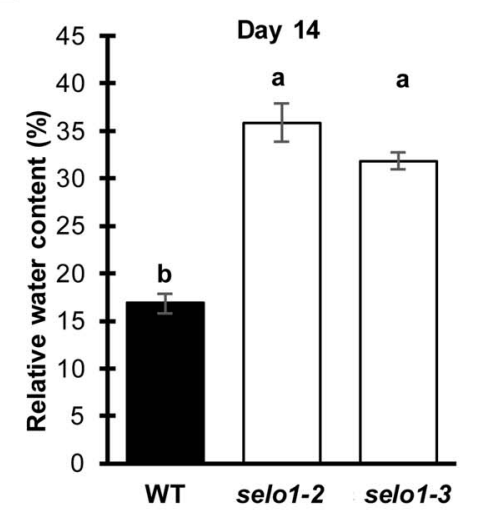

I

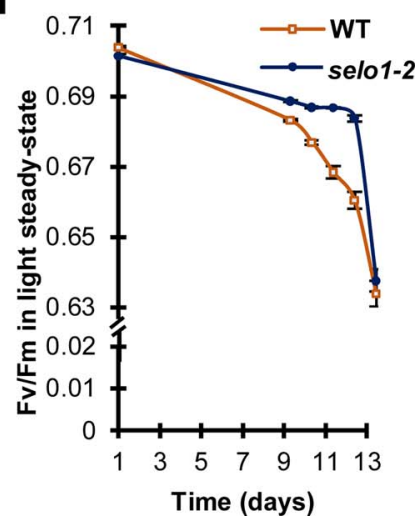

Fig. 1. Absence of SELO increases dehydration tolerance.

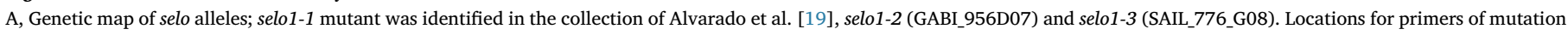

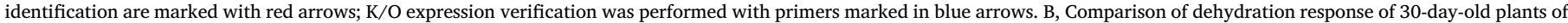

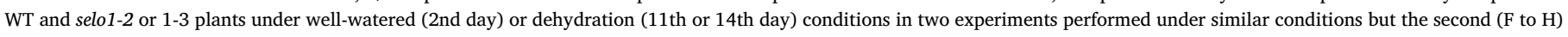

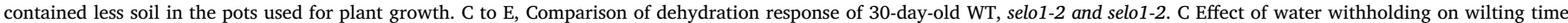

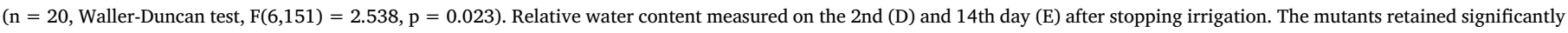

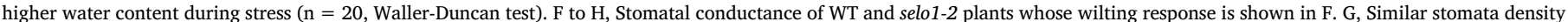

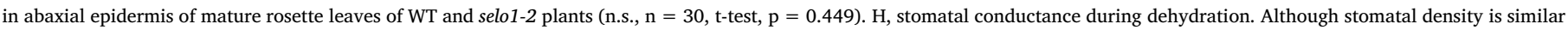

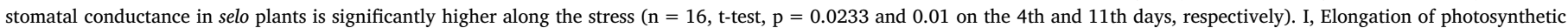

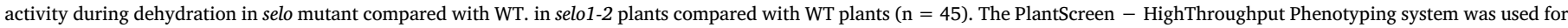
measuring of 30-day-old plants $(n=45)$ subjected to water withholding in the experiment shown in $F$.

from the public collections of T-DNA insertion mutants, which carry TDNA insertions in intron 1, and isolated homozygous mutant lines. In each homozygous mutant, the T-DNA insertion site and lack of transcription downstream to this site were verified by PCR followed by sequencing and RT-PCR, respectively, using primers indicated in Fig. $1 \mathrm{~A}$ and detailed in Table A1.

According to public compilations of transcript profiling data, SELO expression mainly occurs in photosynthetic tissues including rosette and cauline leaves, and flower sepals. In rosette leaves SELO expression is upregulated during early stages of drought, cold and heat stresses and late stages of osmotic and UVB stresses (Fig. A1 and Fig. A2). Expression of other already identified Arabidopsis selenoprotein-like proteins is not specifically stimulated by different abiotic stresses as is demonstrated by analysis performed using the Genevestigator site (Fig. 
A3, Table A2, [57]).

Because transcription of ProDH1 is inhibited in photosynthetic tissues by water deficit similarly to salt stress $[17,58]$, we performed dehydration assays with soil-grown homozygous selo mutants comparing them with WT plants. Under normal growth conditions phenotypic features and development of the selo mutants were indistinguishable from WT plants and no significant differences were observed in all rosette morphological parameters which were examined in experiments shown in Fig. 1B, and Fig. A4, and demonstrated in Fig. A5 . However, when exposed to dehydration, wilting of selo1-2 and selo1-3 mutants was delayed by about 1 to 3 days compared to WT, as demonstrated in three different kinds of dehydration experiments (Fig. 1B and F and Fig. A4 D).

Augmentation of dehydration tolerance in selo mutants was confirmed by monitoring leaf relative water content (RWC) close to wilting time. Whereas on day 2 following water withdrawal selo and WT plants displayed similar leaf RWC (Fig. 1D), both selo1-2 and selo1-3 mutants retained about 2-fold higher leaf RWC on day 14 of dehydration compared to WT (Fig. 1E). When water content of total rosette was estimated, both selo1-2 and selo1-3 had higher rosette water content during dehydration compared with WT plants (Fig. A4G). Although the number of stomata per leaf area was similar in leaves of WT and mutant plants (Fig. 1G, t-test, $\mathrm{p}=0.449$ ), leaf stomatal conductance in the selo1-2 mutant was higher than that of WT plants (Fig. 1H), during normal (Day 4, t-test, $\mathrm{p}=0.023$ ) and dehydration (Day 11, t-test, $\mathrm{p}=0.01$ ) conditions. These surprising results suggested that the tolerance to water deficit in selo1 plants is not directly correlated with stomatal closure as was also observed in Arabidopsis bam1 mutant, which lacks beta amylase activity in chloroplasts of guard cells [59].

Photosynthetic activity was longer maintained in selo mutants compared with WT plants during dehydration. Several photosynthetic parameters were estimated, including PSII efficiency under steady state illumination (Fig. 1I), electron transport rate (ETR; [37]; Fig. A6), and Chlorophyll Fluorescence Decrease ratio $\left(\mathrm{R}_{\mathrm{Fd}}\right)$, which is linearly correlated with photosynthetic $\mathrm{CO}_{2}$ fixation ([38], Fig. A4F). At the beginning of water withholding period there were no difference between $\mathrm{WT}$ and selo plants in Fv/Fm in steady state actinic light and $\mathrm{R}_{\mathrm{Fd}}$. values indicating similar activity of PSII and PSI leading to equal rate of $\mathrm{CO}_{2}$ fixation (Fig. 1I and Fig. A4 E, F). ETR measurement showed that WT has a significantly higher ETR at high irradiation (PAR in the range of 1500 to $3000 \mu$ mole $\mathrm{m}^{-2} \mathrm{~s}^{-1}$ ) at the beginning of the dehydration period (Fig. A6). On the 21th day of dehydration, when dehydrated WT plants had only $60 \%$ water content relatively to irrigated WT plants, photosynthesis activity started to decay in WT plants (Fig. 4A,E and F) with gradual reduction of $\mathrm{Fv} / \mathrm{Fm}$ in steady state light, reflecting the decrease in activity of both PSs, and also leading to diminishing of $\mathrm{CO}_{2}$ fixation. This decline occurred much later in the mutants (Fig. 4A,E and F). They could maintain photosynthetic activity longer than WT plants (Fig. 1I and Fig A4 E), with corresponding delayed decrease of $\mathrm{R}_{\mathrm{Fd}}$ (Fig. 4F) and equal decline of ETR (Fig. A6) compared to WT plants. The extended photosynthetic capacity during dehydration, likely delayed the cellular shortage of photosynthates, NADPH and ATP in the selo mutants during critical dehydration stages.

\subsection{Inactivation of selo alleviates stress-induced proline accumulation}

In Arabidopsis and many other plants, dehydration and salt stress result in transcriptional activation of P5CS1 ( $\triangle(1)$-PYRROLINE-5-CARBOXYLATE SYNTHASE 1) and simultaneous silencing of PRODH1 (PROLINE DEHYDROGENASE 1) genes of the Glu-Pro-Glu cycle (Fig. 2A), stimulating Pro accumulation [8,9,12,17]. The observed capability of selo mutants to grow in the presence of the toxic proline analogue T4C predicted an enhancement of T4C degradation by Pro catabolic pathway. In fact, we observed that free Pro levels remained unchanged in the selo1-2 and selo1-3 mutants ( $t$-test, $\mathrm{p}>0.05)$ compared to 1.5-fold elevation of Pro accumulation in WT between days 2 and 8 of dehydration treatment (Fig. 2B). Quantitative real-time PCR (qRT-PCR) measurements of transcript levels (Fig. 2C) revealed 10- and 5-fold increases of P5CS1 and P5CS2 transcript levels, respectively, between days 2 and day 8 of dehydration in both selo1-2 mutant and WT, indicating similar regulation of genes involved in the first ratelimiting step of Pro synthesis. Transcript levels of P5CR ( $\triangle(1)$-PYRROLINE-5-CARBOXYLATE REDUCTASE) showed about 2-fold lower increase in the selo1-2 mutant compared to WT suggesting a lower enhancement of second step of Pro synthesis. As expected, dehydration stress reduced the expression in WT of PRODH1, catalyzing the first step of Pro degradation. By contrast, $P R O D H 1$ transcription showed over 10fold induction by dehydration, correlating with enhanced T4C tolerance and lack of Pro accumulation in the selo1-2 mutant. In addition, dehydration-induced silencing of ProDH2, the PRODH1 paralogue, did not occur in selo1-2 mutant. Furthermore, transcript levels of $P 5 C D H$ $(\Delta$ (1)-PYRROLINE-5-CARBOXYLATE DEHYDROGENASE) catalyzing the second step of proline degradation increased to about 3-fold higher level compared to WT in the selo1-2 mutant during dehydration. Together, these data confirmed that inactivation of SELO results in inverse regulation of ProDH1 and ProDH2 transcription during dehydration, leading to enhanced mitochondrial Pro degradation and thereby eliminating stress-induced Pro accumulation. Notably, in WT plants, Pro degradation is normally increased only during the recovery period after the stress, by induction of ProDH and P5CDH expression [16].

\subsection{SELO is localized to the chloroplast}

Thus far, it is unknown whether in animal cells SELO as a mitochondrial protein has any effect on regulation of Pro synthesis, catabolism and related ROS generation. While the animal enzymes of Pro synthesis and degradation are localized to mitochondria, in higher plants P5CS enzymes are located in the cytosol and partly in chloroplasts [14]. However, similarly to animal cells and yeast, Pro degradation by PRODH and P5CDH occurs in plant mitochondria, linked to ROS generation by PRODH delivery of electrons to the mitochondrial electron transport chain (METC; [8]). Elevated ROS production induced by different processes, including abiotic stresses, photorespiration, and certain pathogen infections, appears to differentially regulate the expression of $P 5 C S$ and $P R O D H$ genes in Arabidopsis [10,14]. Considering the effect of SELO deficiency on Pro catabolism in plant mitochondria and the mitochondrial location of human SELO, it was intriguing to characterize the cellular location of Arabidopsis SELO and its possible role in ROS regulation.

For examining SELO cellular localization, an expression cassette of SELO-GFP driven by the CaMV 35 S promoter was cloned in pART27 [31] and introduced into selo mutants, resulting in SELO-GFP overexpressing plants. Ectopically expressed SELO-GFP was localized to chloroplasts in leaves (Fig. 3A-H, and Fig. A7), in contrast to mitochondrial location of HsSELO in human cells [25]. To further confirm this observation, SELO-GFP was co-expressed in plants with a chloroplast marker (tpFNR-YFP), composed of N-terminal chloroplast transit peptide of Ferredoxin NADP ${ }^{+}$Reductase (FNR) fused to YFP (received from Prof. Jaideep Mathur). Confocal microscopy imaging of leaf tissues confirmed co-localization of chlorophyll A, SELO-GFP, and tpFNRYFP in chloroplasts (Fig. 3A-H) by fluorescence overlap in chloroplasts in the merged images (white chloroplasts in Fig. 3D and $\mathrm{H}$ ) with a Pearson correlation coefficient of 0.76 . However, presumably due to ectopic expression driven by the constitutive $35 \mathrm{~S}$ promoter, both SELOGFP and tpFNR-YFP were also detected in the cytosol around the large vacuole of leaf cells (Fig. 3B-D). In addition, confocal imaging of ectopically expressed SELO-GFP or tpRbcS-GFP (transit peptide of RUBISCO small subunit fused to GFP) in leaves showed similar chloroplast localization of the two proteins (Fig. A7). To additionally prove chloroplast localization of SELO, samples of total proteins and purified chloroplasts prepared from WT and SELO-GFP seedlings, were resolved by SDS-PAGE and subjected to western blotting with anti-GFP and anti- 

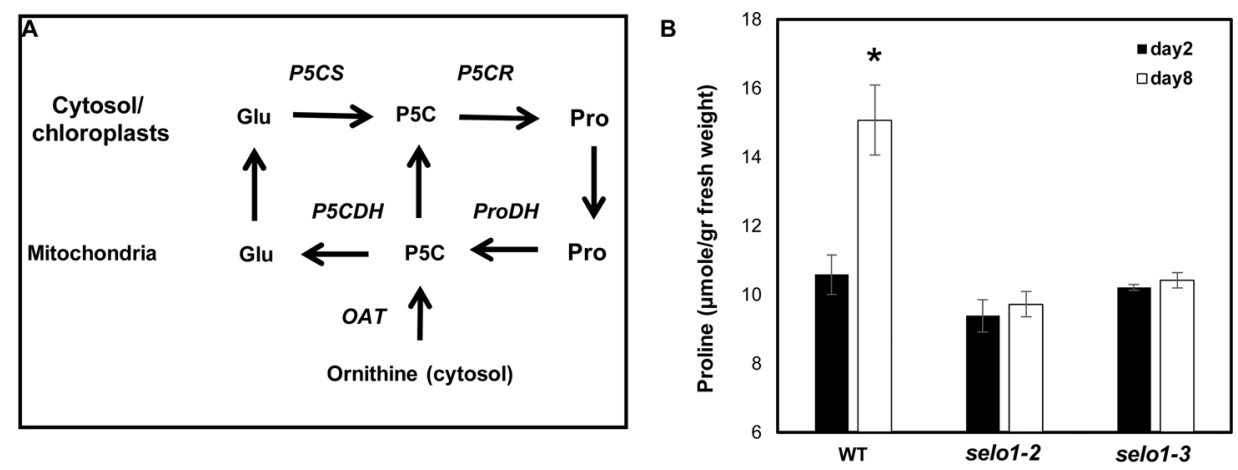

C

WT

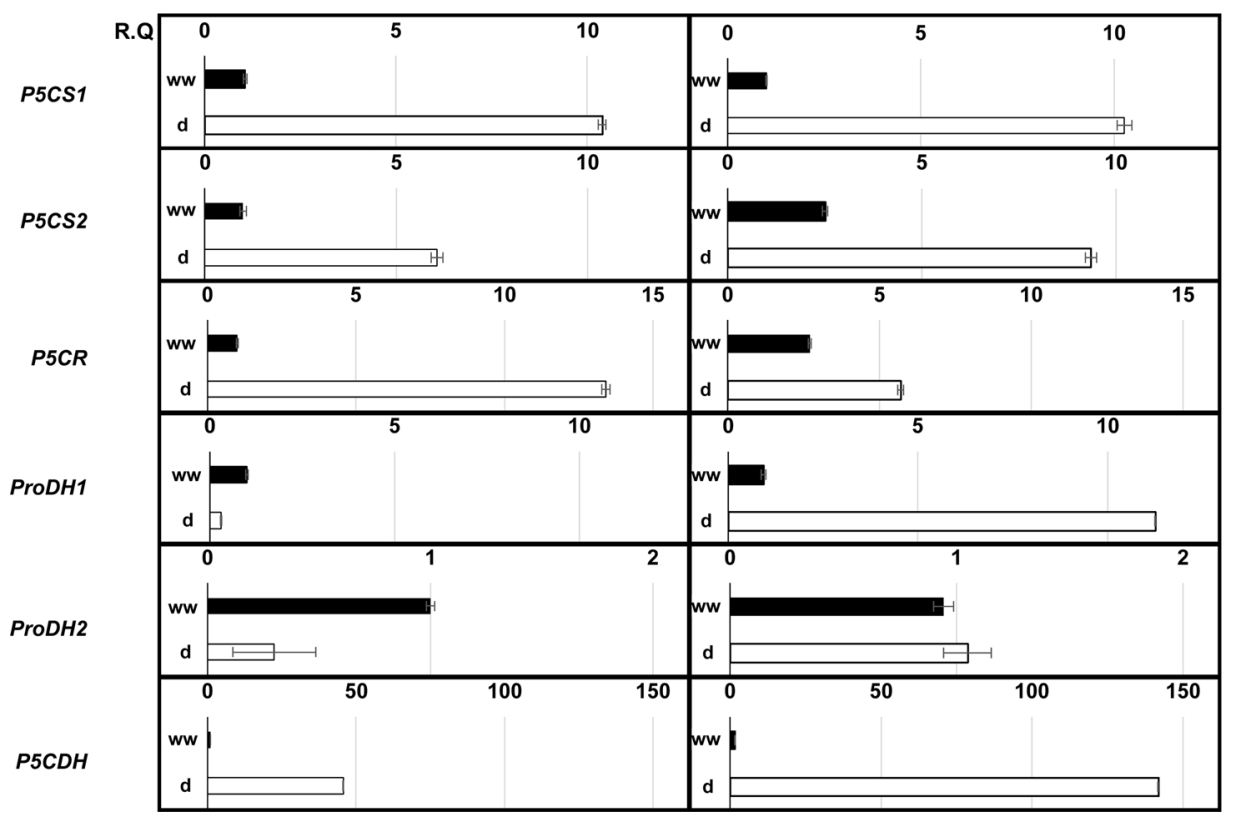

Fig. 2. Low accumulation of proline in selo1-2 mutant coincides with elevated transcript levels of proline degradation enzymes during dehydration.

A, Glutamate-proline-glutamate cycle. Enzymes involved in proline synthesis in the cytosol and likely in chloroplasts or breakdown in mitochondria are indicated.

B, Comparison of proline content after 2 days (ww well watered) and 8 days ( $\mathrm{d}$ - dehydrated) of water withholding in leaves of WT $(\mathrm{n}=10$, t-test, $\mathrm{p}=0.0243)$, selo1-2 $(\mathrm{n}=10$, t-test, $\mathrm{p}=0.298)$ and selo1-3 ( $\mathrm{n}=10$, $\mathrm{t}$-test, $\mathrm{p}=0.377$ ) shows no stressinduced elevation of Pro.

C, Transcript levels of genes encoding enzymes of the glutamate-proline-glutamate cycle in leaves of WT and selo1-2 plants, after 2 days (ww) and 8 days (d) of water withholding. Each qPCR reaction was performed with 3 biological replicates and 3 technical replicates. Transcript levels are relative to the value of well-watered WT. P5C - $\Delta(1)$ - pyrroline-5-carboxylate, P5CS1 and $2-\Delta(1)-$ pyrroline-5-carboxylate synthase 1 and 2, P5CR $-\Delta(1)$-pyrroline-5carboxylate reductase, $\mathrm{ProDH} 1$ and 2 - proline dehydrogenase, $P 5 C D H-\Delta(1)$-pyrroline-5-carboxylate dehydrogenase.
TUBULIN antibodies, using a parallel probing with anti-RUBISCO large subunit (RbcL) antibodies as a control (Fig. 3I). Whereas RbcL was detected in all samples, TUBULIN was present only in total cell extracts verifying the purity of chloroplast preparations. The SELO-GFP signal was detected in extract of total protein and isolated chloroplasts of SELO-GFP seedlings confirming its chloroplast localization.

Next, protein complexes from purified leaf chloroplasts of SELO-GFP plants were resolved on blue-native (BN) gels and subjected to parallel western blotting with anti-GFP antibody, which revealed their cofractionation with SELO-GFP (Fig. 3J). The detected bands of protein complexes (left panel) were excised and subjected to LC-MS/MS analysis to determine their major components (Table A3). The microsequencing results indicated that SELO-GFP occurred in association with relatively fast migrating thylakoid complexes, enriched in PSII components, cytochrome b6f and few PSI components (Fig. 3J, complexes in bands 5, 6, 8, and 9, which had high score values in Table A3). These findings are in agreement with previously reported large-scale chloroplast proteomics studies, which predicted that SELO carries an Nterminal chloroplast transit peptide of 66 amino acids and is present in chloroplast stromal fraction $[60,61]$. An additional step was taken by conducting co-immunoprecipitation (Co-IP) analysis, using anti-GFP antibody conjugated to agarose beads (GFP-Trap, Chromotek) with partially dissolved complexes from isolated leaf chloroplasts of SELOGFP or WT plants. Firstly, microsequencing of total pulled down fractions was conducted, in which FNR was identified as the main interactor of SELO-GFP compared with similar analysis of WT fraction. Thereafter, Co-IP fractions were resolved by SDS-PAGE and following blotting, were probed with antibodies recognizing representatives of electron acceptors downstream to PSI (Fig. 3K). Interactions with FNR (35 kDa) (LFNR1 and LFNR2 are recognized by the antibodies) [62,63], and PGRL1A (AT4G22890; Protein Proton Gradient Regulation 5-Like 1A) were identified. PGRL1 and PGR5 form a complex that controls the major pathway of cyclic electron flow (CEF) in the vicinity of PSI [64,65]. It is not clear whether in C3 plants PGR5-PGRL1 complex competes with FNR on reduced Fd (Ferredoxin), or also interacts with it to mediate CEF and linear electron flow (LEF) activity [66]. Since both FNR and PGRL1A were recently reported as being phosphorylated by STN7 or its paralog STN8, respectively [67], we examined probing with anti-STN7 antibodies, and detected interaction of SELO-GFP with STN7 kinase (Fig. 3K).

The latter interaction implies that SELO may have a broad influence, since STN7 is the major kinase that phosphorylates LHC2, FNR, and few other chloroplast proteins [67]. Furthermore, STN7 activity was found to be controlled by the redox state of the plastoquinone pool [3], and therefore PSII activity responsible for plastoquinone reduction might also be involved. STN7 kinase activity indirectly mediates electron flow through PSI and downstream by being the major phosphorylating agent of LHCII thereby enabling its movement to form LHCII/LHCI/PSI the detected interactions indicate that SELO might participate in controlling the balance between CEF and LEF activities [68]. Nonetheless it should be noted that further characterization of SELO interactions is needed order to assess its regulatory role and verify the involvement of its putative redox motif and kinase domain [26]. 

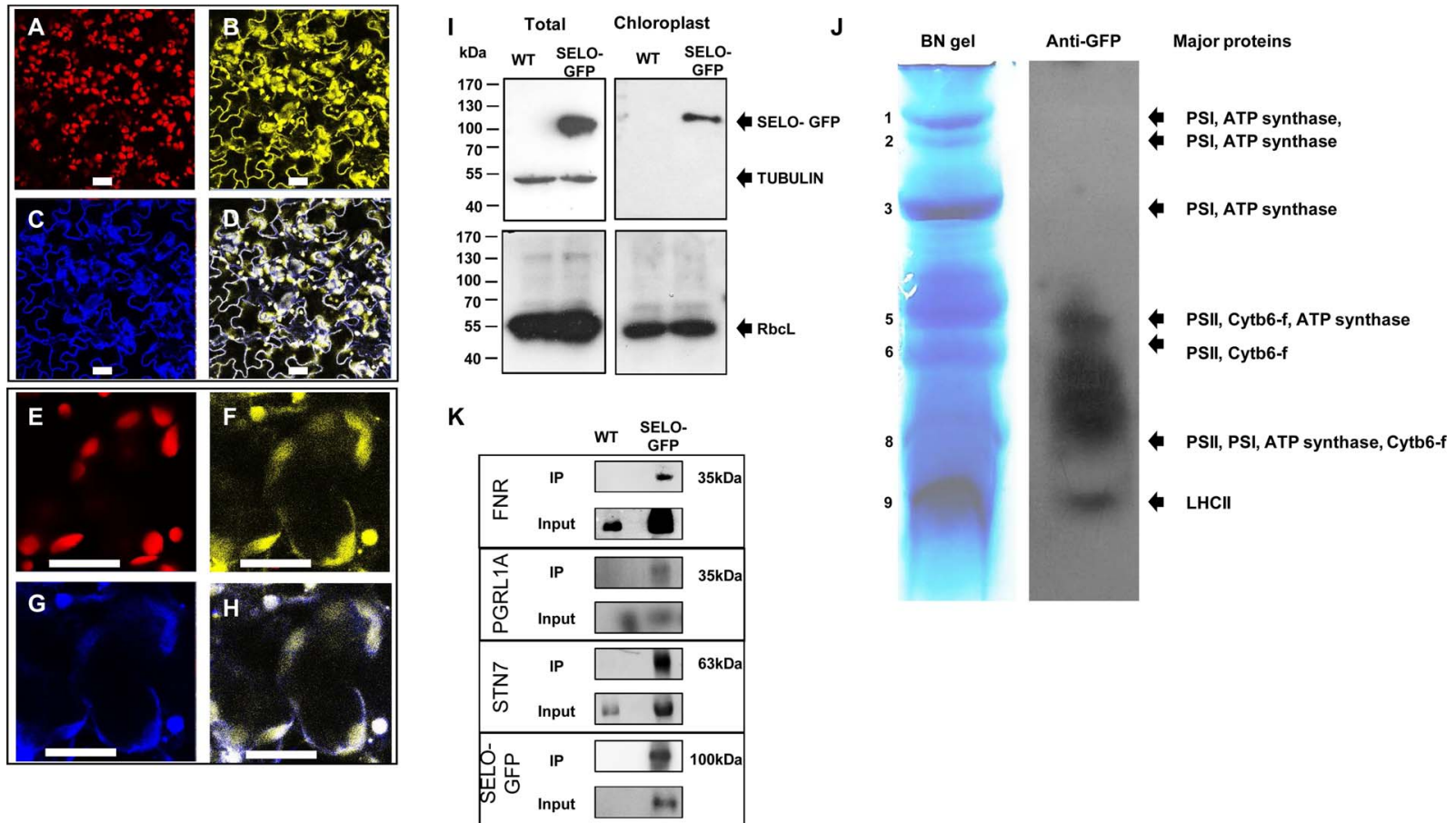

Fig. 3. SELO is localized to chloroplasts.

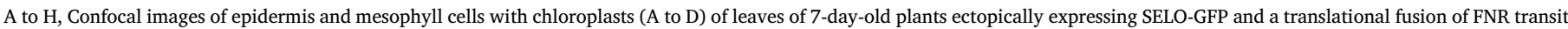

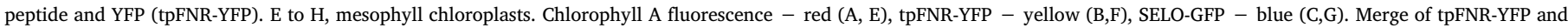
SELO-GFP images (D, H). Co-localization of the marker fluorescence appears as white. Bars correspond to $10 \mu \mathrm{m}$ in all images.

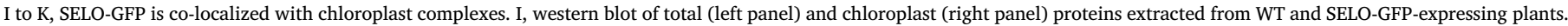

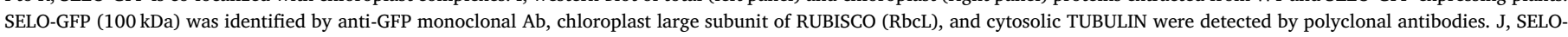

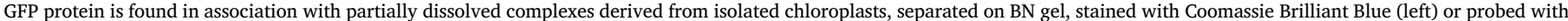

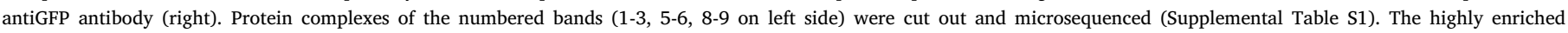

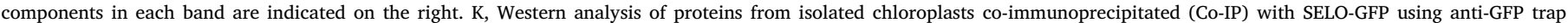
indicates interactions with proteins in PSI vicinity. Input - chloroplast proteins prior to Co-IP, IP - GFP-SELO interacting proteins identified by specific antibodies.

\subsection{Inactivation of SELO lowers ROS levels}

It is well-documented that, in addition to the reduction of $\mathrm{NADP}^{+}$, Ferredoxin-Thioredoxin Reductase (FTR) and CEF components, about $10 \%$ of PSI electron flux is directed to $\mathrm{O}_{2}$ by Fd-mediated generation of superoxide radical $\left(\mathrm{O}_{2}^{-}\right.$), which is converted to $\mathrm{H}_{2} \mathrm{O}_{2}$ by $\mathrm{Cu} / \mathrm{Zn}$ Superoxide Dismutase (Cu/Zn-SOD) under normal growth conditions $[3,69]$. Upon drought stress, ABA-signaling stimulates stomatal closure, lowering $\mathrm{CO}_{2}$ availability and fixation rate [70]. Consequent over-reduction of photosynthetic electron transport components enhances production of $\mathrm{O}_{2}^{\bar{c}}$ and $\mathrm{H}_{2} \mathrm{O}_{2}$ at PSI and PQ (plastoquinone), as well as singlet oxygen $\left({ }^{1} \mathrm{O}_{2}\right)$ generation by PSII, which together impose oxidative stress $[11,71]$. According to the notion that guard cell chloroplasts are a major source of cellular ROS production [72], we analyzed changes in fluorescence of the ROS indicator dye $2^{\prime}, 7^{\prime}$-dichlorofluorescin diacetate (DCF) in chloroplasts of stomatal guard cells of leaves in both WT and selo1-2 plants, in response to increased light intensity (Fig. 4A and B). Confocal analysis (Fig. 4A) and quantification of DCF fluorescence (Fig. 4B) indicated that ROS content of selo1-2 chloroplasts was about $50 \%$ of that of WT under normal irradiation $\left(100 \mu\right.$ mole $\left.\mathrm{m}^{-2} \mathrm{~s}^{-1}\right)$. Upon enhancing ROS production by exposing plants to 2-fold higher light intensity for $30 \mathrm{~min}$ (HLI in Fig. 4A and B), the DFC fluorescence values still remained about $20 \%$ lower in the selo1-2 mutant compared to WT indicating reduced ROS accumulation. Estimation of whole-seedling content of $\mathrm{H}_{2} \mathrm{O}_{2}$, the most stable ROS compound in plant tissues [4], showed that 7-day-old selo1-2 seedlings contained $50 \%$ less $\mathrm{H}_{2} \mathrm{O}_{2}$ relative to WT seedlings (Fig. 4C), suggesting that lower levels of ROS in selo1-2 mutant is likely a whole plant phenomenon.

To stimulate ROS production at PSI, 7-day-old selo1-2 and WT seedlings, cultured for $48 \mathrm{~h}$ in $0.5 \mathrm{MS}$ liquid medium, were treated with $0.25 \mu \mathrm{M}$ methyl viologen (MV, paraquat). Oxidative stress-induced decline of PSII quantum yield (Fv/Fm) was monitored for $3 \mathrm{~h}$ by automatic chlorophyll fluorescence measurement [73,74]. Despite the exerted partial hypoxia due to the experimental conditions of immersing the seedlings in MS medium and gentle shaking, which likely lowered the amount of ascorbate and glutathione content and thus concomitantly decreased $\mathrm{H}_{2} \mathrm{O}_{2}$ quenching by redox enzymes of the waterwater cycle [75,76], we could observe the effect of MV-induced ROS production. MV treatment stimulated a continuous decline of PSII quantum yield in WT (Fig. 4D), which was bigger than that observed in selo1-2 seedlings. Higher $\mathrm{F}_{\mathrm{v}} / \mathrm{F}_{\mathrm{m}}$ values in the mutant both before and during MV-treatment indicated that selo1-2 mutation could lower the impact of MV-induced oxidative stress on PSII activity, likely by elevating antioxidant capacity. Therefore, the level of general oxidant quenching capacity was measured using Trolox equivalent antioxidant capacity (TEAC) assay [45]. Oxidant-quenching capacity in leaves of 30-day-old well-watered WT plants was lower than that of selo1-2 and selo1-3 mutants (Fig. 5B, left panel).

Together, the data indicated that absence of SELO lowers ROS levels and increases oxidant quenching under normal unstressed conditions, or when plants are exposed to MV or high light intensity.

\subsection{Upregulation of genes involved in ROS scavenging by the selo mutation and dehydration effect}

Correlation between higher oxidant scavenging capacity (Fig. 5B) of the selo mutant and its reduced sensitivity to MV raised the possibility that lack of SELO might elevate levels of ROS scavenging enzymes which are numerous in plants [77]. Therefore, we decided to focus on 
A
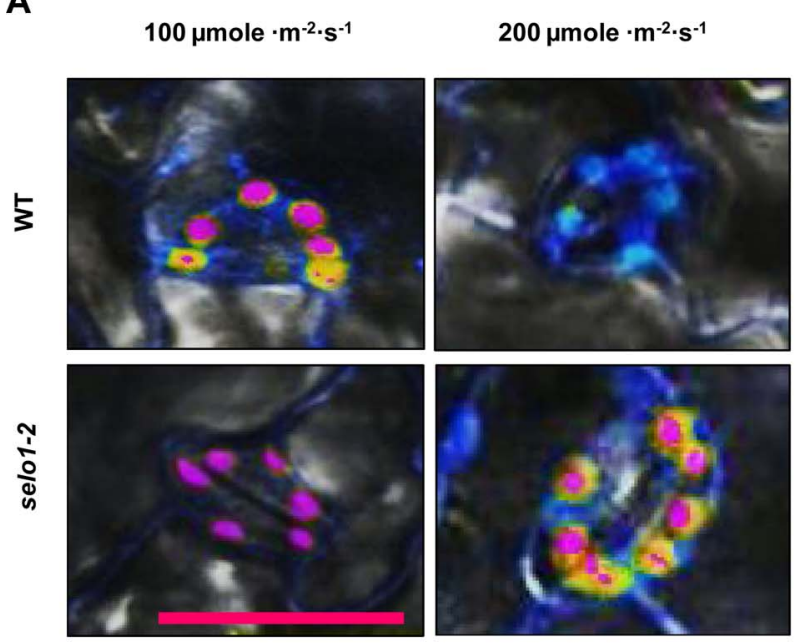

C

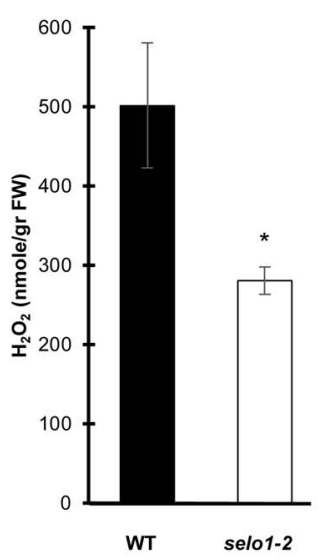

D
B
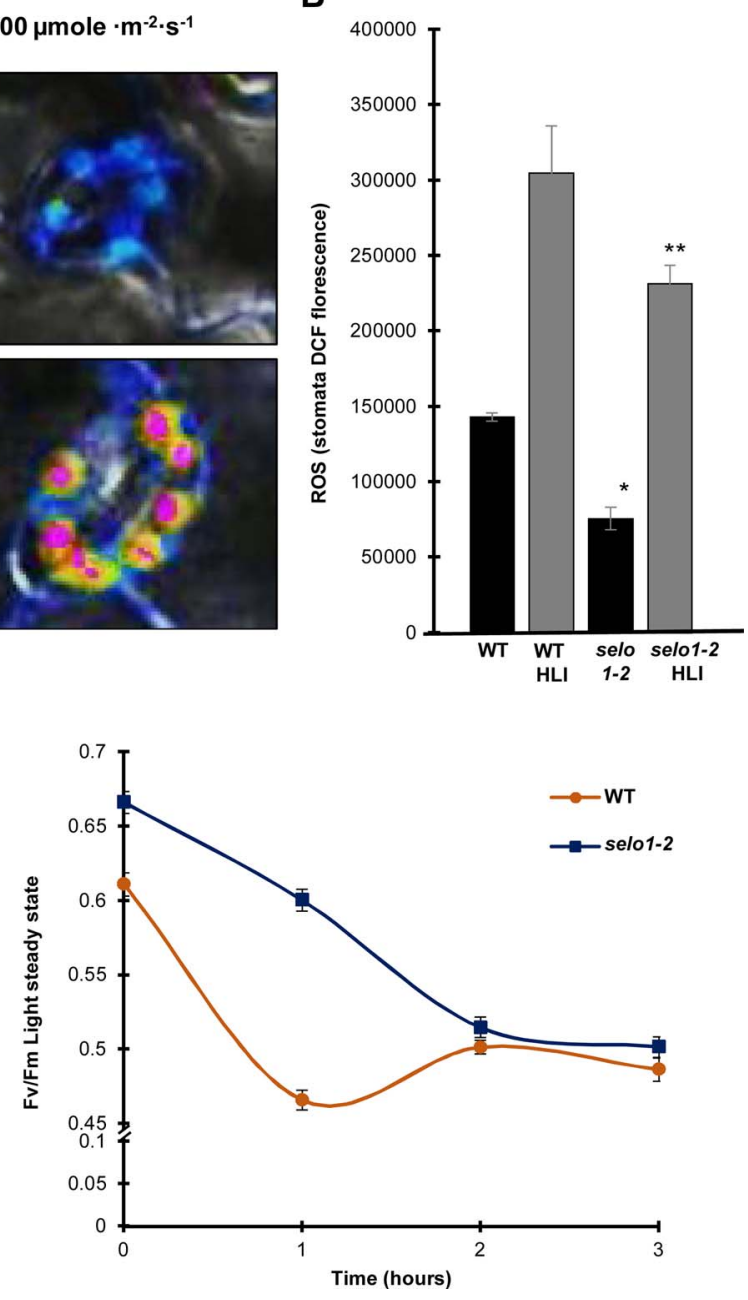

Fig. 4. Absence of SELO alleviates ROS effects.

A and B, Mutants of SELO contain less ROS under normal and light-stress conditions. A, Hydrogen peroxide content in stomata guard cells of WT and selo1 plants exposed to normal irradiation $\left(100 \mu \mathrm{mole} \cdot \mathrm{m}^{-2} \cdot \mathrm{s}^{-1}\right)$ or double light intensity (HLI, $200 \mu$ mole $\cdot \mathrm{m}^{-2} \cdot \mathrm{s}^{-1}$ ) estimated by using DCF dye. DCF fluorescence is in displayed in blue and that of chlorophyll A in magenta. B, Hydrogen peroxide levels in chloroplasts, shown in A, quantified using IMAGE J. Hydrogen peroxide levels in WT plants significantly exceeded those of selo1 ( $\mathrm{n}=10$, Mann-Whitney-Wilcoxon test). Bar corresponds to $20 \mu \mathrm{m}$ in all images. C, Quantification of hydrogen peroxide levels in 7-day-old WT and selo1-2 seedlings grown in liquid MS medium. Oxidation of ADHP dye in extracts of seedlings vs. calibration curve of $\mathrm{H}_{2} \mathrm{O}_{2}$ revealed higher levels in WT than in selo1-2 $(\mathrm{n}=5$, $\mathrm{t}$-test, $\mathrm{t}=0.05)$. D, ROS produced by the PSI electron acceptor methyl viologen affect PSII: Seven-day-old WT and selo1-2 seedlings were incubated in 0.5 MS medium with methyl viologen $(0.25 \mu \mathrm{M})$ for 5 hours in wells of a micro-titter plate $(n=24)$. The MV effect was assessed by comparing photosystem II efficiency (Fv/Fm) using PlantScreen HighThroughput Phenotyping system. evaluating transcript levels of representative enzymes involved in scavenging of ROS produced by MV effects, photorespiration and Pro catabolism.

MV competes with Fd as an efficient electron acceptor from $F_{A} / F_{B}$ of PSI. Thus, lower oxidative damage of PSII in the selo mutant (Fig. 4D) suggested that lack of SELO could enhance scavenging of superoxide anions $\left(\mathrm{O}_{2} \cdot{ }^{-}\right)$. Several steps are involved in scavenging of PSI-produced $\mathrm{O}_{2} \cdot{ }^{-}$. Thylakoid $\mathrm{Cu} / \mathrm{Zn}$-SOD enzyme (CSD2) dispropotionates $\mathrm{O}_{2} \cdot{ }^{-}$to $\mathrm{H}_{2} \mathrm{O}_{2}$, which is then reduced to water by the thylakoid Ascorbate Peroxidase (tAPX). $\mathrm{O}_{2} \cdot{ }^{-}$released to the stroma is quenched by Fe-SODs and stromal APX (sAPX). Peroxiredoxins (PRXRs), including peroxiredoxin Q (PRXQ), present in thylakoid lumen and stromal side of thylakoid membranes, reduce $\mathrm{H}_{2} \mathrm{O}_{2}$ after being reduced either by FTR-TRX or NADPH-NTRC pathways. Scavenging of ROS generated by the PSIIover-reduction of the PQ pool is performed separately, and partially involved Plastid Terminal Oxidase (PTOX), [3,71,78,79].

We estimated the transcript levels of nuclear genes encoding representatives of ROS quenching enzymes by qRT-PCR measurements. Under well-watered (WW) conditions, leaves of 30-days-old plants revealed 1.5 to 2-fold higher expression, in the selo1-2 mutant compared with WT, of the nuclear genes CDS2, FDS3 (Fe-SOD), tAPX, sAPX, PRXQ, GR2 (Glutathione Reductase 2) and MDAR6 (MonoDehydro-Ascorbate Reductase 6) encoding chloroplast ROS scavenging enzymes (Fig. 5A). GR2 was reported to play an important role in protecting PSII from $\mathrm{H}_{2} \mathrm{O}_{2}$ damage [80]. Less is known about MDAR6, present in both chloroplast stroma and mitochondria, and likely has a broad spectrum of substrates, even being capable of generating superoxide from the explosive 2,4,6-trinitrotoluene (TNT, [81]).
Interestingly MSD1 encoding the mitochondrial Mn-SOD also showed upregulation in unstressed selo1-2 mutant, similarly to chloroplast CSD2 and FSD2. However, WT and selo plants displayed comparable expression of $A O X 1 A$ encoding mitochondrial alternative oxidase under WW conditions (Fig. 5). AOX1A activity is considered as a key regulator of mitochondrial-chloroplast inter-organelle signaling [82]. Its inactivation results in enhanced ROS damage of chloroplast photosynthetic apparatus, conferring acute sensitivity to drought and light stress [83]. In contrast, transcription of plastid terminal oxidase PTOX and the prokaryotic-type thioredoxin TRXm2, involved in chloroplast PSII biogenesis and in interactions with mitochondrial and peroxisome/chloroplast targeted proteins, showed lower expression in the selo1-2 mutant under WW conditions. Other genes encoding key enzymes of ROS inactivation, the peroxisomal CATALASE 2 (CAT2) and cytoplasmic APX2, had similar transcript levels in WT and selo1-2 plants under WW conditions, similarly to $R B O H D$ gene encoding plasmamembrane NADPH oxidase, which is involved in ROS signaling related to osmotic stress, ABA, salicylic acid and pathogen attacks. In summary, higher expression of genes encoding key enzymes of chloroplast ROS scavenging by SOD/ascorbate/glutathione cycle correlated with lower ROS levels and elevated oxidant scavenging capacity in selo mutant compared with WT plants (Figs. 4 and 5).

Next, we examined how imposition of dehydration stress affects the transcript levels of these genes encoding ROS scavenging enzymes. qRTPCR measurements were performed with similarly grown WT and selo12 plants subjected to 8 days of dehydration. In contrast to well-watered conditions, transcript levels of CDS2, FDS3, sAPX, and PRXQ genes were extremely reduced in selo1-2 similarly to WT plants (Fig. 5A, Appendix 


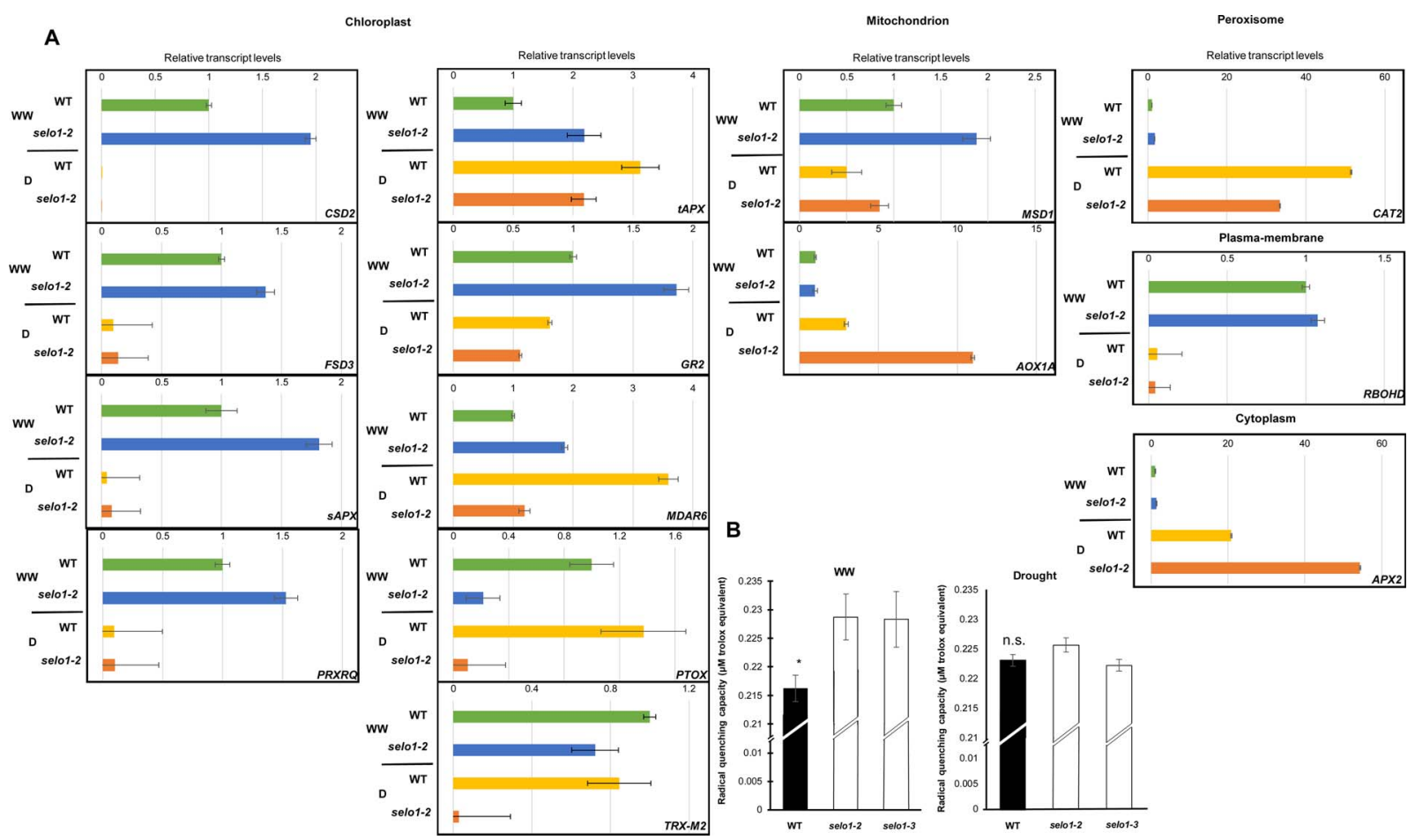

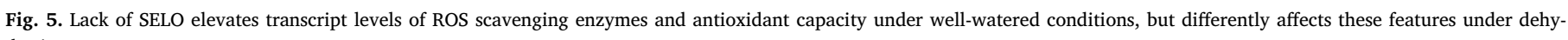
dration stress.

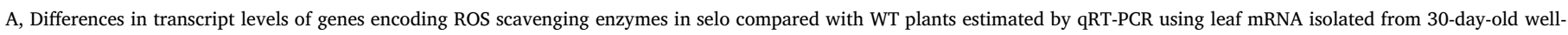

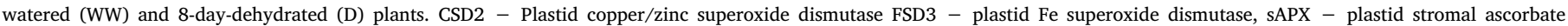

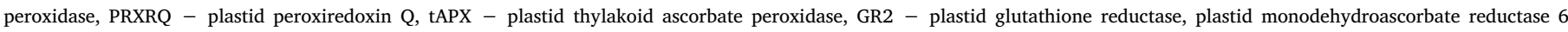

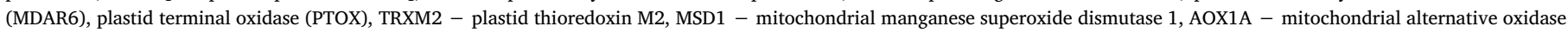

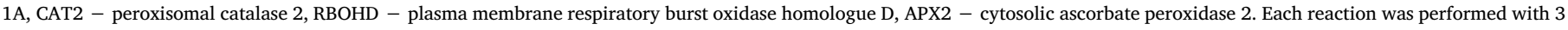
biological replicates and 3 technical replicates. Transcript levels are relative to the value of well-watered WT. Estimated values are listed in Table A4.

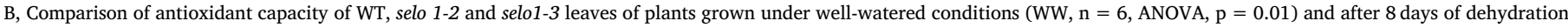

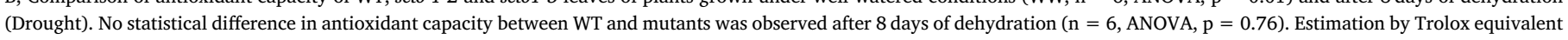
antioxidant capacity (TEAC) assay.

Table A4), indicating that scavenging of PSI-derived $\mathrm{O}_{2} \cdot{ }^{-}$might be significantly lower under dehydration conditions.

Transcript levels of enzymes participating in chloroplast glutathione-ascorbate cycle were higher than those directly involved in $\mathrm{O}_{2} \cdot{ }^{-}$elimination. Transcription of $t A P X$ and MDAR6 was induced in WT during dehydration but not in selo1-2, while transcript levels of GR2 showed only slight decline in selo1-2 compared to WT under water shortage.

The PTOX/AOX4 gene encoding the thylakoid alternative Ubiquinol:Oxygen Oxidoreductase showed lower expression in the selo1-2 mutant compared to WT, with and without dehydration. Transcript levels of the TRXm2, a representative of the FTR-TRX system, were notably reduced due to dehydration in selo1-2 compared to WT.

A drop in mitochondrial MSD1 mRNA levels occurred in both WT and selo mutant during dehydration, while the expression of another mitochondrial $\mathrm{O}_{2} \cdot{ }^{-}$scavenger the alternative oxidase $A O X 1 A$ was notably increased to about 3-fold in WT and 10-fold in selo1-2 plants. Higher expression of $A O X 1 A$ thus correlated with the induced expression of $\mathrm{PrODH}$ and $\mathrm{P} 5 \mathrm{CDH}$ genes of ROS-producing, mitochondrial Pro degradation pathway in the selo1-2 mutant (Fig. 2C). Transcript levels of cytosolic APX2 and peroxisomal CAT2 were very low in both WT and selo mutant under WW conditions, and significantly elevated during dehydration. APX2 transcript levels were 2.6-fold higher but CAT2 levels were about 2-fold lower in selo1-2 compared to WT during dehydration. Finally, $R B O H D$ expression was repressed in both WT and selo1-2 plants by dehydration (Fig. 5, Appendix Table A4).

These data revealed that nearly all chloroplast ROS scavenging functions, except for $t A P X$, were down-regulated in the selo1-2 mutant during dehydration at similar or higher magnitude compared to WT.
Nonetheless, in correlation with de-repression of genes of mitochondrial Pro degradation during dehydration (Fig. 2), two important ROS scavenging functions defined by mitochondrial AOX1A and cytosolic $A P X 2$ were transcriptionally upregulated in the selo1-2 mutant during stress. AOX1A and APX2 belong to a group of co-regulated genes encoding chloroplast and mitochondrial redox proteins, which are activated by WRKY63 and repressed by WRKY40 transcription factors during ABA-dependent responses to high light and abiotic stress $[84,85]$.

Considering the influence of additional factors related to ROS scavenging capacity, such as post translational changes, regulation of enzymatic activity, high abundance of additional ROS scavenging enzymes [77], and cellular content of non enzymatic oxidant quenching compounds [71], we also compared the total oxidant scavenging capacity of WT and selo plants under well-watered and dehydration conditions (Fig. 5B). While at the onset of dehydration, the total antioxidant capacity of selo mutants compared with that of WT was $6 \%$ higher, this difference disappeared during dehydration by the decline in selo mutants and elevation in WT of antioxidant capacity. Consequently the measured antioxidant capacity of selo mutants and WT plants was similar, with no significant difference, on the 8th day of dehydration (Fig. 5B, right panel). Nonetheless, the differential transcription pattern of specific genes, e.g. APX2, AOXA, PTOX, MDAR6 and TRX-M2, in selo plants during dehydration compared with WT (Fig. 5A) implies that SELO absence changes the regulation of ROS scavenging not only under well watered conditions but also during dehydration stress. 


\section{Discussion}

\subsection{Changes linked to SELO's absence}

We identified plant SELO in a forward genetics approach designed to detect knockout mutants with disturbed abiotic stress signaling. Characterization of selo mutants demonstrated changes in ROS content and in transcript levels of nuclear encoded ROS scavenging enzymes most of them targeted to the chloroplast. Upon imposition of dehydration, selo mutants were more tolerant to the stress, and revealed disturbed Pro metabolism. The following sections are centered towards elucidating the linkage between the observed changes occurring in the selo mutants towards characterizing SELO role in plants.

\subsection{Selenoprotein $O$ and other selenoprotein-including systems}

Evolutionary studies have identified SELO proteins as highly conserved in all three domains of life and suggested that their ancient role was linked to aquatic/anaerobic life that existed well before the appearance of terrestrial organisms (Fig. A8 ; $[20,86]$ ). SELO homologues have a conserved putative redox-active motif, implying their involvement in thiol-disulfide exchange redox reactions. Human SELO (HsSELO; $73.4 \mathrm{kDa}$ ) was localized to mitochondria and found capable of forming a complex of about $88 \mathrm{kDa}$ under oxidative conditions. This SELO complex was more stable when the resolving second Cys residue was exchanged to Ser in the C-terminal redox motif (SxxC). The identity of redox-dependent interacting partner of about approximately $14 \mathrm{kDa}$ has remained unknown [25]. Other classes of mammalian selenoproteins are functioning as glutathione peroxidases (GPXs), thioredoxin reductases (TRXRs) and deiodinases (DIOs) [87]. In addition to SELO, two other mammalian selenoproteins, TRX-reductases (TR2 and 3) and glutathione peroxidase (GPX4) are also found in mitochondria and participate in control of redox homeostasis [25,88,89].

Dudkiewicz et al. [26] identified putative kinase-related signatures, which are conserved in all SELO proteins. These domains are part of the designated YdiU/UPF0061 family of unknown function according to NCBI database. The name of this domain refers to the E. coli SELO homologue YdiU, which is transcriptionally activated by IscR, a key redox regulator of $\mathrm{Fe}-\mathrm{S}$ cluster (Isc) biogenesis and other genes encoding Fe-S proteins in E. coli and other bacteria [90]. Similarly to bacteria, mRNA and protein levels of numerous human selenoproteins are upregulated in response to oxidative stress [91]. In Arabidopsis, no significant changes in expression of genes encoding selenoprotein-like proteins other than SELO could be detected (Fig. A3), except for the gene encoding mitochondrial GPX6, which is cold-induced.

Selenoproteins contain a SEC residue in their redox motif, however, except for the mitochondrial genome of American cranberry [92], it appears that the selenocysteine insertion machinery (tRNA-Sec and SECIS) was lost during the evolution of higher plants. Compared to bacteria and animals, only few classes of selenoproteins, owning Cys instead of Sec residue in their redox motif, have remained in plants (defined as selenoprotein-like), the rest were eliminated throughout evolution [20]. The Arabidopsis genome includes 10 selenoproteinencoding genes, most of them have been barely characterized [20,86]. In comparison to animal GPX-selenoproteins, playing essential roles in $\mathrm{H}_{2} \mathrm{O}_{2}$ inactivation, the redox regulatory functions of plant GPXs have been less explored [93,94], whereas much attention has been focused on catalases, peroxiredoxins and enzymes of the ascorbate/GSH cycle, considered as the major $\mathrm{H}_{2} \mathrm{O}_{2}$ scavengers in higher plants [71]. Plant selenoprotein-like GPXs are NADPH-dependent enzymes, carrying twoCys-redox motifs, and structurally related to animal TRX reductases, the functions of which are replaced in plants by NADPH-TRX reductases, including chloroplast NADPH-TRX Reductase C (NTRC).

Two chloroplast systems control the shift of redox equivalents from PSI to TRXs and peroxiredoxins; the FTR/TRX pathway starting with ferredoxin-TRX reductases (FTRA and FTRB) that accept electrons from
Fd, and FNR-NTRC pathway in which FNR-reduced NADPH is used by NTRC to reduce peroxiredoxins and to lesser extent TRXs. Both systems display different TRX substrate specificity with certain overlapping $[3,79]$. NTRC, FTRs, as well as GRXs, were reported to reduce peroxiredoxins, which inactivate a broad range of peroxide-containing substrates [77]. Chloroplast GPXs, such as GPX1 and 7 in Arabidopsis, are thought to prefer lipid peroxide substrates and are reduced by TRXs (reviewed by Dietz [95]). Notably, Arabidopsis cytosolic GPX3 was found to interact with, oxidize and thereby inhibit the activity of PP2C phosphatase ABI2 (ABA INSENSITIVE 2), resulting in activation of ABAsignaling, reduced water loss and enhanced drought tolerance [96]. This illustrates that plant selenoprotein-like proteins can potentially play a role in modulating the redox status of some other proteins, in addition to their well-known substrates.

\subsection{Plant SELO is localized to chloroplasts and interacts with proteins related to $P S I$}

Our study has provided experimental evidence (Fig. 3) that SELO is a chloroplast protein in Arabidopsis. SELO-GFP was localized to chloroplasts by confocal microscopy and by western analysis of proteins from purified chloroplasts. It was found in association with partially solubilized thylakoid-membrane protein complexes following BN gel separation and immunoblotting. Co-immunoprecipitation using antiGFP antibody and isolated chloroplasts revealed direct interactions of SELO-GFP with FNR, PGRL1A and STN7, which did not occur in chloroplasts isolated from WT plants. These interactions imply SELO involvement in LEF and CEF downstream to PSI [3,77].

SELO was previously identified as unknown protein in the chloroplast stromal fraction by large-scale proteomic studies, as well as by bioinformatics identification of proteins with predicted chloroplast transit peptides (At_CHLORO database; $[60,61]$ ). The N-terminal region, comprising chloroplast transit peptide in plant and algal SELO homologues, shows no sequence similarity to animal counterparts. Our subcellular localization data also show SELO-GFP presence in the cytosol, which is likely the consequence of ectopically expressing SELOGFP driven by the strong constitutively active CaMV 35S promoter. Cterminal GFP fused to human SELO did not prevent its mitochondrial localization in human cells [25]. However, despite our efforts, we could not find so far evidence for mitochondrial localization of SELO in any tissue. Database searches indicated that SELO mRNA levels are higher in leaves and other photosynthesizing tissues and elevated by oxidative and abiotic stress stimuli, including drought, salt stress, ozone and UV irradiation (Fig. A1 and A2).

\subsection{Implication of SELO in regulation of ROS production}

Initial characterization of Arabidopsis selo mutants indicated that absence of SELO decreases ROS levels in seedlings, and more specifically in chloroplasts of guard cells by 50\% (Fig. 4A and B). It also lowers ROS production and oxidative damage of PSII, which is induced by high light or methyl-viologen (MV). As MV competes with ferredoxin (Fd) accepting electrons from $\mathrm{F}_{\mathrm{A}} / \mathrm{F}_{\mathrm{B}}$ components of PSI reaction center, this observation suggests that SELO might be involved in modulating the elimination of PSI-produced superoxide anions [3]. Fd controls the redox status of Fe-S components in PSI reaction centers by transferring electrons to Fd-NADP + reductase (FNR), Fd-Txr reductase (FTRA and FTRB), and Fd-plastoquinone reductases suggested to be the PGR5PGRL1A and NDH complexes [64,65,97]. However, Fd cannot secure full redox protection of PSI reaction centers under various intensities of illumination, thus a significant portion of electron flux is directed to $\mathrm{O}_{2}$ thereby producing superoxide radicals $\left(\mathrm{O}_{2}^{\overline{2}}\right)$, even under optimal photosynthetic conditions $[75,78,98]$. In co-immunoprecipitation experiments of chloroplast complexes with SELO-GFP, SELO was found to interact with FNR and PGRL1 (Fig. 3K), which are Fd-dependent electron acceptors involved in LEF and CEF respectively [3,65], confirming 
the prediction of SELO involvement in sensing or mediating both types of electron flows. SELO also interacted with STN7 but not with its paralogue STN8, or with Fd. STN7 is a thylakoid membrane kinase required for the photosynthetic transition from state 1 to state 2 . This transition is dependent on LHCII phosphorylation by STN7, controlled by the redox state of the plastoquinone (PQ) pool and its interaction with Cytb $_{6} f[99,100]$. STN7 possesses two two-Cys redox motifs, one of them is thought to be a target of TRXf and is situated in the ATP binding pocket, likely responsible for STN7 inactivation under high light intensity (HLI). This motif is absent in STN8 in correlation with its insensitivity to HLI [101]. Studies comparing stn7 mutant with WT plants demonstrated that STN7 is also involved in phosphorylation of FNR and RbcL assuming their accessibility to the thylakoid-assembled STN7 [67]. Considering the detected SELO interactions, indicating its contact with PSI components, still it could well be presumed that other proteins might interact with SELO and therefore additional studies are required to elucidate the functional role of SELO in processes downstream to PSI.

\subsection{Transcriptional upregulation of chloroplast ROS scavenging functions}

Our data demonstrated lower $\mathrm{H}_{2} \mathrm{O}_{2}$ levels and higher oxidant scavenging capacity in selo mutant grown under normal conditions (Figs. 4C and 5B, respectively), which might be an outcome of enhanced expression of genes that encode ROS scavenging proteins. Elevation of transcript levels of nuclear encoded ROS scavenging enzymes associated with PSI activity was observed in selo mutant grown under normal growth conditions (Fig. 5A, WW). The observed increase encompasses chloroplast redox network starting with the superoxide scavengers copper/zinc superoxide dismutase (CDS2) and its stromal counterpart Fe-SOD (FSD2), as well as the second line of $\mathrm{H}_{2} \mathrm{O}_{2}$ scavengers of thylakoid and stromal ascorbate peroxidases ( $t A P X$ and $S A P X)$, and thylakoid-bound peroxiredoxin (PRXQ). It further included other components of the redox circles, such as glutathione reductase (GR2) and mono-dehydroacorbate reductase (MDAR6) genes [4]. In addition, the transcript level of mitochondrial manganese-SOD was also elevated, indicating superoxide elevated scavenging also in mitochondria.

This concerted elevation seems to be unique, because according to transcript profiling data derived from the Genevestigator site (https:// genevestigator.com [57]), the overall expression of these genes is not stimulated by any of the measured redox changes or in any reported mutant, not even by MV treatment that generates superoxide. However, downregulation by drought [83] and hypoxia was observed, except for tAPX and MDAR6 [102]. Reports related to single enzymes showed that ectopic overexpression of $T A P X$ and $S A P X$ could confer MV tolerance [103] and protection against high light-induced oxidative stress [104]. Mutation of GR2 could elevate high light sensitivity and increase ROS production [80], whereas PRXQ activity was shown to be involved in protection of photosystems and chloroplast redox homeostasis $[105,106]$. Thus, this general elevation of ROS scavenging capacity in the absence of SELO likely indicates a kind of general alarm sensed in PSI vicinity under unstressed growth conditions.

Nonetheless, during exposure to dehydration stress a change in this elevation phenomenon was observed, since the enhancement disappeared and either similar or lower expression of certain genes could be observed in the selo mutant compared to WT (Fig. 5, "D" treatment). The transcript abundance of enzymes related to the glutathione-ascorbate cycle, such as tAPX, MDAR6 and GR2 was lower during dehydration in the selo mutant compared with WT. Hence, while under normal growth conditions high expression of ROS scavenging enzymes correlated with higher capacity of ROS elimination, the change in transcript abundance data of dehydrated selo and WT plants suggests that the regular stress-mediated control of these genes is more effective in silencing the transcription of superoxide scavenging enzymes in PSI vicinity, which can be thought as less needed considering the gradual lowering of ETR during stress (Fig. A6). Nevertheless lack-of-SELO effect during dehydration stimulates the expression of cytoplasmatic $A P X 2$ and mitochondrial AOX1, shifting the influence outside the chloroplast where it is likely needed for ROS scavenging due to elevation of Pro catabolism that delivers electrons to mitochondrial ETC $[8,10]$. Correlatively with the changes in transcription profiles of genes encoding ROS scavenging enzymes in the selo mutant during dehydration stress, the recorded total antioxidant capacity in the mutant was similar to WT, due to the observed raise in WT and decrease in selo mutants of antioxidant capacity relative to the elevated levels in the mutant before the stress (Fig. 5B).

Although an overall transcription profiling of ROS related enzymes linked to proteomics is still required for better understanding of ROS scavenging elevation in the absence of SELO, the current data suggest that the longer survival of selo mutants and their extended photosynthesis ability, compared with WT during dehydration, is likely not a direct consequence of general elevation of antioxidant capacity. Thus it maybe speculated that the dehydration withstanding of selo mutants is likely an outcome of a shift to better energy provision in mitochondria by Pro catabolism that delivers electrons to the mitochondrial electron chain [107] while utilizing NADPH produced in chloroplasts for maintaining proline synthesis and $\mathrm{NADP}^{+}$provision.

\subsection{Unusual physiological phenomena correlate with perturbed signaling in selo mutants}

Certain irregular events recorded in selo mutants under unstressed or/and stress conditions demonstrate differences in signaling caused by lack of SELO:

The selo mutants are less sensitive to dehydration, compared with WT plants. They show a delay of about 3 days in wilting time, which is associated with extended photosynthetic activity. Correlatively, selo mutants maintain higher water content relative to WT plants under dehydration conditions, and by contrast reveal higher stomatal conductance in leaves along the stress. Thus, instead of dehydration-stimulated stomatal closure, which is controlled in WT plants mainly by ABA- and ROS-mediated signaling [69,70,108,109], it seems that lack of SELO modulates a different signaling scenario that allows stomata opening and maintaining of higher water content under dehydration conditions. Albert et al. [70], have summarized the presently available data on regulation modes responsible for stomatal closure, illustrating a network of 84 cellular components having 156 interactions among them. Therefore, many more parameters related to stomatal closure have to be evaluated in order to predict the signaling mode that differentially controls stomata in selo mutants.

The response of the selo mutant to dehydration does not include net increase in proline content despite the always mentioned concept that considers proline as an osmo-protectant, scavenger of ROS and essential metabolite for rendering plants tolerant to abiotic and biotic stresses $[13,14,110,111]$. Nevertheless, the role of proline in elevating salt and drought-stress tolerance is still under debate since increase in Pro is not always correlated with stress resistance $[10,15]$. Moreover, stress-induced elevation of Pro synthesis that utilizes NADPH may change NADP + to NADPH ratio, allow continuous photosynthesis in chloroplasts, enhance oxidative pentose phosphate pathway and provide mitochondria with redox equivalents produced in chloroplasts. Therefore, the mentioned metabolic effects of Pro may be considered more beneficial under stress conditions rather than the properties of the Pro molecule $[9,10]$. Upon exposure to dehydration selo mutant shows disturbed Pro catabolism signaling that leads to increased expression instead of silencing of ProDH1 and ProDH2, encoding the first enzyme in Pro degradation, accompanied by an elevation of $P 5 C D H$ transcription. Consequently, no raise in Pro content occurred despite the increase in Pro synthesis.

In response to inhibition of photosynthesis and glucose limitation, Pro degradation pathway is upregulated by AMP-activated kinases in mammals and their SnRK1 homologues in plants [112,113]. 
Enhancement of mitochondrial proline degradation channels electrons via FAD-containing ProDH to ubiquinone in the mitochondrial electron transport chain in both plant and human cells [114-116]. We have previously shown that the Pro degradation intermediate P5C largely enhances ROS production in the absence of P5CDH [8]. Enhanced transcription of $P 5 C D H$ gene in the selo mutant thus likely eliminates P5C accumulation. At the same time, about 3-fold higher expression of AOX1A gene of cyanide-resistant mitochondrial alternative oxidase suggests higher level of activation of so-called non-energy conserving pathway in the selo mutant, which through the inter chloroplast-mitochondrion malate/oxaloacetate shuttle lowers the accumulation of chloroplast NADPH, and thereby PSI photoinhibition [117,118].

Consequently, this mechanism also suggests that the primary signal affected by the selo mutation is related to the NADPH level, which reflects the redox status of Fd in the chloroplast. Recently, transcriptional activation of ProDH1 promoter was shown to be repressed by chemical inhibition of NADPH oxidases and treatment of plants with the ROS scavengers ascorbic acid (AsA) and N,N'-dimethylthiourea (DMTU). In addition, two mutations inactivating Cytochrome P450 (CYP86A2) and Long Chain Acyl Synthetase2 (LACS2), which catalyze two successive steps in very-long-chain fatty acid (VLCFA) synthesis, were found to activate the ProDH1 promoter under unstressed conditions [119]. These mutations do not prevent ProDH1 inactivation by low water potential, while in the selo mutant ProDH silencing is cancelled during dehydration. However, examples of ProDH transcription regulation by diverse factors such as VLCFAs, which are synthesized in the endoplasmic reticulum and involved in phospholipid and sphingolipid signaling [120], or lack of SELO that also increases nuclear transcription of ROS scavenging enzymes under unstressed conditions, raise the possibility that SELO is likely involved in redox regulation of transcription factors. Such direct and indirect regulation as reviewed by Dietz [121], still requires further unraveling of signaling components that explain the linkage between SELO presence in PSI vicinity and its exerted control of redox status.

\section{In summary}

The thylakoid-associated SELO has been studied by analyzing physiological events derived from its absence in the selo mutant. The recorded events include elevated dehydration tolerance with extended functioning of photosynthetic photosystems, and low levels of ROS under unstressed conditions linked to increased transcript levels of nuclear encoded ROS scavenging enzymes acting mostly in chloroplasts. Specific elevation of proline degradation occurs in mitochondria of selo mutant during dehydration due to unique increase in transcription of ProDHs and P5CDH. Studying genome-wide transcriptional changes in the selo mutants, further identification of redox partners and examining the involvement of predicted kinase domain and redox motif of SELO should provide additional insights about SELO regulatory functions.

\section{Accession numbers}

AOX1A - AT3G22370; CAT2 - AT4G35090; CSD2 - AT2G28190; Cyclophilin - AT2G36130; FSD3 - AT5G23310; GR2 - AT3G54660; MDAR6 - AT1G63940; MSD1- AT3G10920; P5CDH - AT5G62530; P5CR - AT5G14800; P5CS1 - AT2G39800; P5CS2 - AT3G55610; PRODH1 - AT3G30775; PRODH2 - AT5G38710; PRXQ AT3G26060; PTOX - AT4G22260; RBOHD - AT5G47910; sAPX AT4G08390; SELO - AT5G13030; tAPX - AT1G77490; TRX-M2 AT4G03520

\section{Acknowledgements}

We thank Jaideep Mathur (University of Guelph; Guelph, Canada) for the seeds of plants overexpressing tpFNR-YFP, Shimon Gepstein
(The Technion; Haifa, Israel) for the anti RbcL antibodies and Anne Harzen (MPI for Plant Breeding Research; Cologne, Germany) for expert assistance in the mass spectrometry analyses. The advice and assistance of Haviva Eilenberg, Guilia Meshulam, Silvia Schuster and Chen Suchariano (Tel Aviv University, Tel Aviv, Israel) is greatly appreciated. We thank Dario Leister and Thilo Ruehle (Ludwig-MaximiliansUniversity, Munich, Germany) for their advices and antibody provision, and Iftach Yacoby and Pini Marcu (Tel Aviv University, Tel Aviv, Israel) for anti-FNR antibodies. T-DNA insertion line - GABI_956D07 was generated in the context of the GABI-Kat program and provided by Bernard Weisshaar (MPI for Plant Breeding Research; Cologne, Germany). LC-MS/MS analyses were performed by the Smoler Proteomics Center, Faculty of Biology, The Technion, Haifa 32000, Israel. The work was funded by DFG German-Israeli-Palestinian Trilateral Program (KO 1438/13-1 to AZ, CK), the Israeli Science Foundation (ISF-1432/08 to AZ), I-CORE Program of the Planning and Budgeting Committee and the Israel Science Foundation (grant no. 757/12 To HF).

\section{Appendix A. Supplementary data}

Supplementary data associated with this article can be found, in the online version, at https://doi.org/10.1016/j.plantsci.2018.02.023.

\section{References}

[1] V. Raja, U. Majeed, H. Kang, K.I. Andrabi, R. John, Abiotic stress: Interplay between ROS, hormones and MAPKs, Environ. Exp. Bot. 137 (2017) 142-157.

[2] F.K. Choudhury, R.M. Rivero, E. Blumwald, R. Mittler, Reactive oxygen species, abiotic stress and stress combination, Plant J. 90 (2017) 856-867.

[3] D. Leister, Piecing the Puzzle Together: the central role of reactive oxygen species and redox hubs in chloroplast retrograde signaling, Antioxid Redox Sign. (2017), http://dx.doi.org/10.1089/ars.2017.7392.

[4] G. Noctor, J.-P. Reichheld, C.H. Foyer, ROS-related redox regulation and signaling in plants Seminars in Cell \& Developmental Biology, (2017), http://dx.doi.org/10. 1016/j.semcdb.2017.07.013.

[5] K. Shinozaki, M. Uemura, J. Bailey-Serres, E.A. Bray, E. Weretilnyk, Responses to abiotic stress, in: B.B. Buchanan, W. Gruissem, R.L. Jones (Eds.), Bichemistruy \& Molecular Biology of Plants, Wiley Blackwell, New Jersey, 2015, p. 1280.

[6] K. Das, A. Roychoudhury, Reactive oxygen species (ROS) and response of antioxidants as ROS-scavengers during environmental stress in plants, Front. Environ. Sci. 2 (2014) 53.

[7] G. Miller, H. Stein, A. Honig, Y. Kapulnik, A. Zilberstein, Responsive modes of Medicago sativa proline dehydrogenase genes during salt stress and recovery dictate free proline accumulation, Planta 222 (2005) 70-79.

[8] G. Miller, A. Honig, H. Stein, N. Suzuki, R. Mittler, A. Zilberstein, Unraveling delta (1)-pyrroline-5-carboxylate-proline cycle in plants by uncoupled expression of proline oxidation enzymes, J. Biol. Chem. 284 (2009) 26482-26492.

[9] P.E. Verslues, S. Sharma, Proline metabolism and Its implications for plant-environment interaction, The Arabidopsis Book, (2010) (e0140).

[10] K. Ben Rejeb, C. Abdelly, A. Savoure, How reactive oxygen species and proline face stress together, Plant Physiol. Biochem. 80 (2014) 278-284.

[11] N. Suzuki, R.M. Rivero, V. Shulaev, E. Blumwald, R. Mittler, Abiotic and biotic stress combinations, New Phytol. 203 (2014) 32-43.

[12] Y. Fichman, S.Y. Gerdes, H. Kovacs, L. Szabados, A. Zilberstein, L.N. Csonka, Evolution of proline biosynthesis: enzymology, bioinformatics, genetics, and transcriptional regulation, Biol. Rev. 90 (2015) 1065-1099.

[13] P.D. Hare, W.A. Cress, Metabolic implications of stress-induced proline accumulation in plants, Plant Growth Regul. 21 (1997) 79-102.

[14] L. Szabados, A. Savoure, Proline: a multifunctional amino acid, Trends Plant Sci. 15 (2010) 89-97.

[15] H. Stein, A. Honig, G. Miller, O. Erster, H. Eilenberg, L.N. Csonka, L. Szabados, C. Koncz, A. Zilberstein, Elevation of free proline and proline-rich protein levels by simultaneous manipulations of proline biosynthesis and degradation in plants, Plant Sci. 181 (2011) 140-150.

[16] X.W. Liang, L. Zhang, S.K. Natarajan, D.F. Becker, Proline mechanisms of stress survival, Antioxid Redox Sign. 19 (2013) 998-1011.

[17] G.B. Bhaskara, T.H. Yang, P.E. Verslues, Dynamic proline metabolism: importance and regulation in water limited environments, Front. Plant Sci. 6 (2015) 484.

[18] J.K. Zhu, Abiotic Stress Signaling and Responses in Plants, Cell 167 (2016) 313-324.

[19] M.C. Alvarado, L.M. Zsigmond, I. Kovacs, A. Cseplo, C. Koncz, L.M. Szabados, Gene trapping with firefly luciferase in Arabidopsis. Tagging of stress-responsive genes, Plant Physiol. 134 (2004) 18-27.

[20] A.V. Lobanov, D.E. Fomenko, Y. Zhang, A. Sengupta, D.L. Hatfield, V.N. Gladyshev, Evolutionary dynamics of eukaryotic selenoproteomes: large selenoproteomes may associate with aquatic life and small with terrestrial life, Genome Biol. 8 (2007). 
[21] R. Brigelius-Flohe, M. Maiorino, Glutathione peroxidases, Bba-Gen Subjects 1830 (2013) 3289-3303

[22] E.S.J. Arner, Focus on mammalian thioredoxin reductases - Important selenoproteins with versatile functions, Bba-Gen Subjects 1790 (2009) 495-526.

[23] L. Schomburg, Selenium, selenoproteins and the thyroid gland: interactions in health and disease, Nat. Rev. Endocrinol. 8 (2012) 160-171.

[24] T.C. Stadtman, Selenocysteine, Annu. Rev. Biochem. 65 (1996) 83-100.

[25] S.J. Han, B.C. Lee, S.H. Yim, V.N. Gladyshev, S.R. Lee, Characterization of mammalian selenoprotein O: a redox-active mitochondrial protein, Plos One 9 (2014) e95518.

[26] M. Dudkiewicz, T. Szczepinska, M. Grynberg, K. Pawlowski, A novel protein kinase-like domain in a selenoprotein, widespread in the tree of life, Plos One 7 (2012) e32138.

[27] D. Leister, L.S. Wang, T. Kleine, Organellar gene expression and acclimation of plants to environmental stress, Front. Plant Sci. 8 (2017) 387.

[28] M.G. Rosso, Y. Li, N. Strizhov, B. Reiss, K. Dekker, B. Weisshaar, An Arabidopsis thaliana T-DNA mutagenized population (GABI-Kat) for flanking sequence tagbased reverse genetics, Plant Mol. Biol. 53 (2003) 247-259.

[29] A. Sessions, E. Burke, G. Presting, G. Aux, J. McElver, D. Patton, B. Dietrich, P. Ho, J. Bacwaden, C. Ko, J.D. Clarke, D. Cotton, D. Bullis, J. Snell, T. Miguel, D. Hutchison, B. Kimmerly, T. Mitzel, F. Katagiri, J. Glazebrook, M. Law, S.A. Goff, A high-throughput Arabidopsis reverse genetics system, Plant Cell 14 (2002) 2985-2994.

[30] K. Yamada, J. Lim, J.M. Dale, H. Chen, P. Shinn, C.J. Palm, A.M. Southwick, H.C. Wu, C. Kim, M. Nguyen, P. Pham, R. Cheuk, G. Karlin-Newmann, S.X. Liu, B. Lam, H. Sakano, T. Wu, G. Yu, M. Miranda, H.L. Quach, M. Tripp, C.H. Chang, J.M. Lee, M. Toriumi, M.M.H. Chan, C.C. Tang, C.S. Onodera, J.M. Deng, K. Akiyama, Y. Ansari, T. Arakawa, J. Banh, F. Banno, L. Bowser, S. Brooks, P. Carninci, Q. Chao, N. Choy, A. Enju, A.D. Goldsmith, M. Gurjal, N.F. Hansen, Y. Hayashizaki, C. Johnson-Hopson, V.W. Hsuan, K. Iida, M. Karnes, S. Khan, E. Koesema, J. Ishida, P.X. Jiang, T. Jones, J. Kawai, A. Kamiya, C. Meyers, M. Nakajima, M. Narusaka, M. Seki, T. Sakurai, M. Satou, R. Tamse, M. Vaysberg, E.K. Wallender, C. Wong, Y. Yamamura, S. Yuan, K. Shinozaki, R.W. Davis, A. Theologis, J.R. Ecker, Empirical analysis of transcriptional activity in the Arabidopsis genome, Science 302 (2003) 842-846.

[31] A.P. Gleave, A versatile binary vector system with a T-DNA organizational-structure conducive to efficient Integration of cloned DNA into the plant genome, Plant Mol. Biol. 20 (1992) 1203-1207.

[32] C. Koncz, J. Schell, The promoter of Tl-DNA gene 5 controls the tissue-specific expression of chimeric genes carried by a novel type of Agrobacterium binary vector, Mol. Gen. Genet. 204 (1986) 383-396.

[33] S.J. Clough, A.F. Bent, Floral dip: a simplified method for Agrobacterium-mediated transformation of Arabidopsis thaliana, Plant J. 16 (1998) 735-743.

[34] M.H. Schattat, S. Griffiths, N. Mathur, K. Barton, M.R. Wozny, N. Dunn, J.S. Greenwood, J. Mathur, Differential coloring reveals that plastids do not form networks for exchanging macromolecules, Plant Cell 24 (2012) 1465-1477.

[35] H. Slatyer, Plant-water relationships, Academic Press, London and New York, 1967.

[36] M. Awlia, A. Nigro, J. Faikus, S.M. Schmoeckel, S. Negrao, D. Santelia, M. Trtilek, M. Tester, M.M. Julkowska, K. Panzarova, High-throughput non-destructive phenotyping of traits that contribute to salinity tolerance in Arabidopsis thatiana, Front. Plant Sci. 7 (2016) 1414.

[37] T. Rungrat, M. Awlia, T. Brown, R. Cheng, X. Sirault, J. Fajkus, M. Trtilek, B. Furbank, M. Badger, M. Tester, B.J. Pogson, J.O. Borevitz, P. Wilson, Using phenomic analysis of photosynthetic function for abiotic stress response gene discovery, The Arabidopsis Book, (2016) (e0185).

[38] H.K. Lichtenthaler, G. Langsdorf, S. Lenk, C. Buschmann, Chlorophyll fluorescence imaging of photosynthetic activity with the flash-lamp fluorescence imaging system, Photosynthetica 43 (2005) 355-369.

[39] C.L. Wilson, P.L. Pusey, B.E. Otto, Plant epidermal sections and imprints using cyanoacrylate adhesives, Can. J. Plant Sci. 61 (1981) 781-783.

[40] K.J. Livak, T.D. Schmittgen, Analysis of relative gene expression data using realtime quantitative PCR and the 2(T)(-Delta Delta C) method, Methods 25 (2001) 402-408.

[41] L.S. Bates, R.P. Waldren, I.D. Teare, Rapid determination of free proline for waterstress studies, Plant Soil 39 (1973) 205-207.

[42] Y. Leshem, L. Seri, A. Levine, Induction of phosphatidylinositol 3-kinase-mediated endocytosis by salt stress leads to intracellular production of reactive oxygen species and salt tolerance, Plant J. 51 (2007) 185-197.

[43] R.A. McCloy, S. Rogers, C.E. Caldon, T. Lorca, A. Castro, A. Burgess, Partial inhibition of Cdk1 in G(2) phase overrides the SAC and decouples mitotic events, Cell Cycle 13 (2014) 1400-1412.

[44] M.J. Zhou, Z.J. Diwu, N. PanchukVoloshina, R.P. Haugland, A stable nonfluorescent derivative of resorufin for the fluorometric determination of trace hydrogen peroxide: Applications in detecting the activity of phagocyte NADPH oxidase and other oxidases, Anal. Biochem. 253 (1997) 162-168.

[45] G. Polturak, N. Grossman, D. Vela-Corcia, Y.H. Dong, A. Nudel, M. Pliner, M. Levy, I. Rogachev, A. Aharoni, Engineered gray mold resistance, antioxidant capacity, and pigmentation in betalain-producing crops and ornamentals, Proc. Natl. Acad. Sci. U. S. A. 114 (2017) 9062-9067.

[46] D. Joly, R. Carpentier, Rapid isolation of intact chloroplasts from spinach leaves, in: R. Carpentier (Ed.), Photosynthesis Research Protocols, Humana Press, Totowa, NJ, 2011, pp. 321-325.

[47] I. Wittig, H.P. Braun, H. Schagger, Blue native PAGE, Nat. Protoc. 1 (2006) 418-428.

[48] E. Zemel, S. Gepstein, Immunological evidence for the presence of ribulose bisphosphate carboxylase in guard-cell chloroplasts, Plant Physiol. 78 (1985) 586-590.

[49] J. Schindelin, I. Arganda-Carreras, E. Frise, V. Kaynig, M. Longair, T. Pietzsch, S. Preibisch, C. Rueden, S. Saalfeld, B. Schmid, J.Y. Tinevez, D.J. White, V. Hartenstein, K. Eliceiri, P. Tomancak, A. Cardona, Fiji: an open-source platform for biological-image analysis, Nat. Methods 9 (2012) 676-682.

[50] S. Pundir, M. Magrane, M.J. Martin, C. O'Donovan, C. The UniProt, Searching and navigating UniProt databases, Current Protocols in Bioinformatics, John Wiley \& Sons Inc., 2002.

[51] K. Katoh, K. Misawa, K. Kuma, T. Miyata, MAFFT: a novel method for rapid multiple sequence alignment based on fast Fourier transform, Nucleic Acids Res. 30 (2002) 3059-3066.

[52] A. Stamatakis, P. Hoover, J. Rougemont, A rapid bootstrap algorithm for the RAxML web servers, Syst. Biol. 57 (2008) 758-771.

[53] G. Fucile, D. Di Biase, H. Nahal, G. La, S. Khodabandeh, Y.N. Chen, K. Easley, D. Christendat, L. Kelley, N.J. Provart, ePlant and the 3D data display initiative: integrative systems biology on the world wide web, Plos One 6 (2011) e15237.

[54] M. Schmid, T.S. Davison, S.R. Henz, U.J. Pape, M. Demar, M. Vingron, B. Scholkopf, D. Weigel, J.U. Lohmann, A gene expression map of Arabidopsis thaliana development, Nat. Genet. 37 (2005) 501-506.

[55] K. Nakabayashi, M. Okamoto, T. Koshiba, Y. Kamiya, E. Nambara, Genome-wide profiling of stored mRNA in Arabidopsis thaliana seed germination: epigenetic and genetic regulation of transcription in seed, Plant J. 41 (2005) 697-709.

[56] J. Kilian, D. Whitehead, J. Horak, D. Wanke, S. Weinl, O. Batistic, C. D'Angelo, E. Bornberg-Bauer, J. Kudla, K. Harter, The AtGenExpress global stress expression data set: protocols, evaluation and model data analysis of UV-B light, drought and cold stress responses, Plant J. 50 (2007) 347-363.

[57] T. Hruz, O. Laule, G. Szabo, F. Wessendorp, S. Bleuler, L. Oertle, P. Widmayer, W. Gruissem, P. Zimmermann, Genevestigator v3: a reference expression database for the meta-analysis of transcriptomes, Adv. Bioinformatics 2008 (2008) 420747.

[58] M. Veerabagu, T. Kirchler, K. Elgass, B. Stadelhofer, M. Stahl, K. Harter, V. MiraRodado, C. Chaban, The Interaction of the Arabidopsis Response Regulator ARR18 with bZIP63 Mediates the Regulation of PROLINE DEHYDROGENASE Expression, Mol. Plant 7 (2014) 1560-1577.

[59] C.M. Prasch, K.V. Ott, H. Bauer, P. Ache, R. Hedrich, U. Sonnewald, Beta-amylase] mutant Arabidopsis plants show improved drought tolerance due to reduced starch breakdown in guard cells, J. Exp. Bot. 66 (2015) 6059-6067.

[60] B. Zybailov, H. Rutschow, G. Friso, A. Rudella, O. Emanuelsson, O. Sun, K.J. van Wijk, Sorting signals, N-terminal modifications and abundance of the chloroplast proteome, Plos One 3 (2008) e1994.

[61] M. Ferro, S. Brugiere, D. Salvi, D. Seigneurin-Berny, M. Court, L. Moyet, C. Ramus, S. Miras, M. Mellal, S. Le Gall, S. Kieffer-Jaquinod, C. Bruley, J. Garin, J. Joyard, C. Masselon, N. Rolland, AT_CHLORO, a comprehensive chloroplast proteome database with subplastidial localization and curated information on envelope proteins, Mol. Cell Proteomics 9 (2010) 1063-1084.

[62] P. Mulo, Chloroplast-targeted ferredoxin-NADP (+) oxidoreductase (FNR): Structure, function and location, Bba-Bioenergetics 2011 (1807) 927-934.

[63] P. Mulo, M. Medina, Interaction and electron transfer between ferredoxin-NADP $(+)$ oxidoreductase and its partners: structural, functional, and physiological implications, Photosynt. Res. 134 (2017) 265-280.

[64] A.P. Hertle, T. Blunder, T. Wunder, P. Pesaresi, M. Pribil, U. Armbruster, D. Leister, PGRL1 is the elusive ferredoxin-plastoquinone reductase in photosynthetic cyclic electron flow, Mol. Cell 49 (2013) 511-523.

[65] W. Yamori, T. Shikanai, Physiological functions of cyclic electron transport around photosystem I in sustaining photosynthesis and plant growth, Annu. Rev. Plant Biol. 67 (2016) 81-106.

[66] M. Suorsa, F. Rossi, L. Tadini, M. Labs, M. Colombo, P. Jahns, M.M. Kater, D. Leister, G. Finazzi, E.M. Aro, R. Barbato, P. Pesaresi, PGR5-PGRL1-dependent cyclic electron transport modulates linear electron transport rate in Arabidopsis thaliana, Mol Plant 9 (2016) 271-288.

[67] A. Schonberg, A. Rodiger, W. Mehwald, J. Galonska, G. Christ, S. Helm, D. Thieme, P. Majovsky, W. Hoehenwarter, S. Baginsky, Identification of STN7/STN8 kinase targets reveals connections between electron transport, metabolism and gene expression, Plant J. 90 (2017) 1176-1186.

[68] T. Shikanai, Regulatory network of proton motive force: contribution of cyclic electron transport around photosystem I, Photosynt. Res. 129 (2016) 253-260.

[69] C.H. Foyer, G. Noctor, Stress-triggered redox signalling: what's in pROSpect? Plant Cell. Environ. 39 (2016) 951-964.

[70] R. Albert, B.R. Acharya, B.W. Jeon, J.G.T. Zanudo, M.M. Zhu, K. Osman, S.M. Assmann, A new discrete dynamic model of ABA-induced stomatal closure predicts key feedback loops, Plos Biol. 15 (2017) e2003451.

[71] G. Miller, N. Suzuki, S. Ciftci-Yilmaz, R. Mittler, Reactive oxygen species homeostasis and signalling during drought and salinity stresses, Plant Cell Environ. 33 (2010) 453-467.

[72] Z. Wang, F.X. Wang, Y.C. Hong, J.R. Huang, H.Z. Shi, J.K. Zhu, Two Chloroplast Proteins Suppress Drought Resistance by Affecting ROS Production in Guard Cells, Plant Physiol. 172 (2016) 2491-2503.

[73] K. Maxwell, G.N. Johnson, Chlorophyll fluorescence - a practical guide, J Exp Bot 51 (2000) 659-668.

[74] A. Krieger-Liszkay, P.B. Kos, E. Hideg, Superoxide anion radicals generated by methylviologen in photosystem I damage photosystem II, Physiol. Plantarum 142 (2011) 17-25.

[75] K. Asada, The water-water cycle as alternative photon and electron sinks, Philos. Trans. Roy. Soc. B 355 (2000) 1419-1430.

[76] A. Paradiso, S. Caretto, A. Leone, A. Bove, R. Nisi, L. De Gara, ROS production an scavenging under anoxia and re-oxygenation in Arabidopsis cells: a balance 
between redox signaling and impairment, Front. Plant Sci. 7 (2016) 1803.

[77] K.J. Dietz, T. Pfannschmidt, Novel regulators in photosynthetic redox control of plant metabolism and gene expression, Plant Physiol. 155 (2011) 1477-1485.

[78] D. Takagi, S. Takumi, M. Hashiguchi, T. Sejima, C. Miyake, Superoxide and singlet oxygen produced within the thylakoid membranes both cause photosystem I photoinhibition, Plant Physiol. 171 (2016) 1626-1634.

[79] K. Yoshida, T. Hisabori, Two distinct redox cascades cooperatively regulate chloroplast functions and sustain plant viability, Proc. Natl. Acad. Sci. U. S. A. 113 (2016) E3967-E3976.

[80] S.H. Ding, R. Jiang, Q.T. Lu, X.G. Wen, C.M. Lu, Glutathione reductase 2 maintains the function of photosystem II in Arabidopsis under excess light, Bba-Bioenergetics 1857 (2016) 665-677.

[81] E.J. Johnston, E.L. Rylott, E. Beynon, A. Lorenz, V. Chechik, N.C. Bruce, Monodehydroascorbate reductase mediates TNT toxicity in plants, Science 349 (2015) 1072-1075.

[82] G.C. Vanlerberghe, G.D. Martyn, K. Dahal, Alternative oxidase: a respiratory electron transport chain pathway essential for maintaining photosynthetic performance during drought stress, Physiol. Plantarum 157 (2016) 322-337.

[83] E. Giraud, L.H.M. Ho, R. Clifton, A. Carroll, G. Estavillo, Y.F. Tan, K.A. Howell, A. Ivanova, B.J. Pogson, A.H. Millar, J. Whelan, The absence of alternative oxidase1a in Arabidopsis results in acute sensitivity to combined light and drought stress, Plant Physiol. 147 (2008) 595-610.

[84] H. Chen, Z.B. Lai, J.W. Shi, Y. Xiao, Z.X. Chen, X.P. Xu, Roles of arabidopsis WRKY18 WRKY40 and WRKY60 transcription factors in plant responses to abscisic acid and abiotic stress, Bmc Plant. Biol. 10 (2010) 281.

[85] O. Van Aken, B.T. Zhang, S. Law, R. Narsai, J. Whelan, AtWRKY40 and AtWRKY63 modulate the expression of stress-responsive nuclear genes encoding mitochondrial and chloroplast proteins, Plant Physiol. 162 (2013) 254-271.

[86] A.V. Lobanov, D.L. Hatfield, V.N. Gladyshev, Eukaryotic selenoproteins and selenoproteomes, Bba-Gen Subjects 1790 (2009) 1424-1428.

[87] V.M. Labunskyy, D.L. Hatfield, V.N. Gladyshev, Selenoproteins: molecular pathways and physiological roles, Physiol. Rev. 94 (2014) 739-777.

[88] A.A. Turanov, D. Su, V.N. Gladyshev, Characterization of alternative cytosolic forms and cellular targets of mouse mitochondrial thioredoxin reductase, J. Biol. Chem. 281 (2006) 22953-22963.

[89] M.A. Reeves, P.R. Hoffmann, The human selenoproteome: recent insights into functions and regulation, Cell Mol. Life Sci. 66 (2009) 2457-2478.

[90] A.D. Nesbit, J.L. Giel, J.C. Rose, P.J. Kiley, Sequence-specific binding to a subset of IscR-regulated promoters does not require IscR Fe-S cluster ligation, J. Mol. Biol. 387 (2009) 28-41.

[91] Z. Touat-Hamici, Y. Legrain, A.L. Bulteau, L. Chavatte, Selective up-regulation of human selenoproteins in response to oxidative stress, J. Biol. Chem. 289 (2014) $14750-14761$.

[92] D. Fajardo, B. Schlautman, S. Steffan, J. Polashock, N. Vorsa, J. Zalapa, The American cranberry mitochondrial genome reveals the presence of selenocysteine (tRNA-Sec and SECIS) insertion machinery in land plants, Gene 536 (2014) 336-343.

[93] K. Bela, E. Horvath, A. Galle, L. Szabados, I. Tari, J. Csiszar, Plant glutathione peroxidases: Emerging role of the antioxidant enzymes in plant development and stress responses, J. Plant Physiol. 176 (2015) 192-201.

[94] G. Passaia, M. Margis-Pinheiro, Glutathione peroxidases as redox sensor proteins in plant cells, Plant Sci. 234 (2015) 22-26.

[95] K.J. Dietz, Thiol-based peroxidases and ascorbate peroxidases: why plants rely on multiple peroxidase systems in the photosynthesizing chloroplast? Mol. Cells 39 (2016) 20-25.

[96] Y.C. Miao, D. Lv, P.C. Wang, X.C. Wang, J. Chen, C. Miao, C.P. Song, An Arabidopsis glutathione peroxidase functions as both a redox transducer and a scavenger in abscisic acid and drought stress responses, Plant Cell 18 (2006) 2749-2766.

[97] J.D. Rochaix, Redox regulation of thylakoid protein kinases and photosynthetic gene expression, Antioxid Redox. Sign. 18 (2013) 2184-2201.

[98] H.P. Misra, I. Fridovich, Generation of superoxide radical during autoxidation of ferredoxins, J. Biol. Chem. 246 (1971) 6886-6890.

[99] P. Pesaresi, A. Hertle, M. Pribil, T. Kleine, R. Wagner, H. Strissel, A. Ihnatowicz, V. Bonardi, M. Scharfenberg, A. Schneider, T. Pfannschmidt, D. Leister,
Arabidopsis STN7 kinase provides a link between short- and long-term photosynthetic acclimation, Plant Cell 21 (2009) 2402-2423.

[100] J.D. Rochaix, Regulation and dynamics of the light-harvesting system, Ann. Rev. Plant Biol. 65 (65) (2014) 287-309.

[101] T. Wunder, W.T. Xu, Q.P. Liu, G. Wanner, D. Leister, M. Pribil, The major thylakoid protein kinases STN7 and STN8 revisited: effects of altered STN8 levels and regulatory specificities of the STN kinases, Front. Plant Sci. 4 (2013) 417.

[102] J.A. Christianson, I.W. Wilson, D.J. Llewellyn, E.S. Dennis, The low-oxygen-induced NAC domain transcription factor ANAC102 affects viability of Arabidopsis seeds following low-oxygen treatment, Plant Physiol. 149 (2009) 1724-1738.

[103] I. Murgia, D. Tarantino, C. Vannini, M. Bracale, S. Carravieri, C. Soave, Arabidopsis thaliana plants overexpressing thylakoidal ascorbate peroxidase show increased resistance to Paraquat-induced photooxidative stress and to nitric oxideinduced cell death, Plant J. 38 (2004) 940-953.

[104] C.H. Pang, K. Li, B.S. Wang, Overexpression of SsCHLAPXs confers protection against oxidative stress induced by high light in transgenic Arabidopsis thaliana, Physiol. Plantarum 143 (2011) 355-366.

[105] P. Lamkemeyer, M. Laxa, V. Collin, W. Li, I. Finkemeier, M.A. Schottler, V. Holtkamp, V.B. Tognetti, E. Issakidis-Bourguet, A. Kandlbinder, E. Weis, M. Miginiac-Maslow, K.J. Dietz, Peroxiredoxin Q of Arabidopsis thaliana is attached to the thylakoids and functions in context of photosynthesis, Plant J. 45 (2006) 968-981.

[106] M. Liebthal, D. Maynard, K.J. Dietz, Peroxiredoxins and Redox Signaling in Plants, Antioxid Redox. Sign. (2017), http://dx.doi.org/10.1089/ars.2017.7164.

[107] C. Cabassa-Hourton, P. Schertl, M. Bordenave-Jacquemin, K. Saadallah, A. Guivarc'h, S. Lebreton, S. Planchais, J. Klodmann, H. Eubel, E. Crilat, D. Lefebvre-De Vos, T. Ghelis, L. Richard, C. Abdelly, P. Carol, H.P. Braun, A. Savoure, Proteomic and functional analysis of proline dehydrogenase 1 link proline catabolism to mitochondrial electron transport in Arabidopsis thaliana, Biochem. J. 473 (2016) 2623-2634.

[108] H. Kollist, M. Nuhkat, M.R.G. Roelfsema, Closing gaps: linking elements that control stomatal movement, New Phytol. 203 (2014) 44-62.

[109] R. Mittler, E. Blumwald, The roles of ROS and ABA in systemic acquired acclimation, Plant Cell 27 (2015) 64-70.

[110] S. Sharma, J.G. Villamor, P.E. Verslues, Essential role of tissue-specific proline synthesis and catabolism in growth and redox balance at low water potential, Plant Physiol. 157 (2011) 292-304.

[111] P.E. Verslues, J.R. Lasky, T.E. Juenger, T.W. Liu, M.N. Kumar, Genome-wide association mapping combined with reverse genetics identifies new effectors of low water potential-induced proline accumulation in Arabidopsis, Plant Physiol. 164 (2014) 144-159.

[112] J. Pandhare, S.P. Donald, S.K. Cooper, J.M. Phang, Regulation and function of proline oxidase under nutrient stress, J. Cell Biochem. 107 (2009) 759-768.

[113] F. Tome, T. Nagele, M. Adamo, A. Garg, C. Marco-Ilorca, E. Nukarinen, L. Pedrotti, A. Peviani, A. Simeunovic, A. Tatkiewicz, M. Tomar, M. Gamm, The low energy signaling network, Front. Plant Sci 5 (2014) 353.

[114] P. Schertl, H.P. Braun, Respiratory electron transfer pathways in plant mitochondria, Front. Plant Sci. 5 (2014) 163.

[115] R.L.S. Goncalves, D.E. Rothschild, C.L. Quinlan, G.K. Scott, C.C. Benz, M.D. Brand, Sources of superoxide/H2O2 during mitochondrial proline oxidation, Redox. Biol. 2 (2014) 901-909.

[116] C.N. Hancock, W. Liu, W.G. Alvord, J.M. Phang, Co-regulation of mitochondrial respiration by proline dehydrogenase/oxidase and succinate, Amino Acids 48 (2016) 859-872.

[117] K. Noguchi, K. Yoshida, Interaction between photosynthesis and respiration in illuminated leaves, Mitochondrion 8 (2008) 87-99.

[118] K.J. Dietz, R. Mittler, G. Noctor, Recent progress in understanding the role of reactive oxygen species in plant cell signaling, Plant Physiol 171 (2016) 1535-1539.

[119] S. Shinde, J.G. Villamor, W.D. Lin, S. Sharma, P.E. Verslues, Proline coordination with fatty acid synthesis and redox metabolism of chloroplast and mitochondria, Plant Physiol. 172 (2016) 1074-1088.

[120] A.D. Du Granrut, J.L. Cacas, How very-long-chain fatty acids could signal stressful conditions in plants? Front. Plant Sci. 7 (2016) 1490.

[121] K.J. Dietz, Redox regulation of transcription factors in plant stress acclimation and development, Antioxid Redox. Sign. 21 (2014) 1356-1372. 